\title{
Membrane Voltage Fluctuations Reduce Spike Frequency Adaptation and Preserve Output Gain in CA1 Pyramidal Neurons in a High-Conductance State
}

\author{
Fernando R. Fernandez, ${ }^{\star}$ Tilman Broicher, ${ }^{\star}$ Alan Truong, and John A. White \\ Department of Bioengineering, Brain Institute, University of Utah, Salt Lake City, Utah 84112
}

\begin{abstract}
Modulating the gain of the input- output function of neurons is critical for processing of stimuli and network dynamics. Previous gain control mechanisms have suggested that voltage fluctuations play a key role in determining neuronal gain in vivo. Here we show that, under increased membrane conductance, voltage fluctuations restore $\mathrm{Na}^{+}$current and reduce spike frequency adaptation in rat hippocampal CA1 pyramidal neurons in vitro. As a consequence, membrane voltage fluctuations produce a leftward shift in the frequencycurrent relationship without a change in gain, relative to an increase in conductance alone. Furthermore, we show that these changes have important implications for the integration of inhibitory inputs. Due to the ability to restore $\mathrm{Na}^{+}$current, hyperpolarizing membrane voltage fluctuations mediated by $\mathrm{GABA}_{\mathrm{A}}$-like inputs can increase firing rate in a high-conductance state. Finally, our data show that the effects on gain and synaptic integration are mediated by voltage fluctuations within a physiologically relevant range of frequencies $(10-40 \mathrm{~Hz})$.
\end{abstract}

\section{Introduction}

Intracellular in vivo recordings in the cortex and hippocampus have established that membrane voltage in neurons in these areas is dominated by intense synaptic activity. These conditions result in large membrane voltage fluctuations and increased membrane conductance (Penttonen et al., 1998; Henze and Buzsáki, 2001; Destexhe et al., 2003; Harvey et al., 2009; Epsztein et al., 2010). Experimental and modeling studies have linked changes in these conditions to the modulation of the sensitivity or gain of the input-output function of individual neurons (Chance et al., 2002; Mitchell and Silver, 2003; Prescott and De Koninck, 2003; Silver, 2010).

An input-output function in neurons can be quantified using a frequency-current $(f-I)$ relationship. Although simple, the tuning and form of this relationship is critical for processing of stimuli and network dynamics (Carandini and Heeger, 1994; Treue and Martínez Trujillo, 1999; Priebe and Ferster, 2008). In vitro work has shown that the slope (gain) of the $f-I$ relationship can be reduced through the introduction of artificially generated membrane voltage fluctuations (Chance et al., 2002; Mitchell and Silver, 2003; Shu et al., 2003). Voltage fluctuations also lead to spike generation at mean voltages below the spike voltage threshold, which enables spike firing to scale with the subthreshold

Received Sept. 28, 2010; revised Jan. 10, 2011; accepted Jan. 14, 2011.

This work was supported by grants from the National Institutes of Health (R01MH085387 and R01MH085074). We thank W. Hamish Mehaffey and Michael N. Economo for useful discussions and comments on the manuscript. *F.R.F. and T.B. share first authorship.

Correspondence should be addressed to either Fernando R. Fernandez or Tilman Broicher, Department of Bioengineering, University of Utah, 20 South 2030 East, 108 BPRB, Salt Lake City, UT 84112, E-mail:f.fernandez@utah.edu or tilman.broicher@utah.edu.

DOI:10.1523/JNEUROSCI.5076-10.2011

Copyright $\odot 2011$ the authors $\quad 0270-6474 / 11 / 313880-14 \$ 15.00 / 0$ membrane voltage (Hô and Destexhe, 2000). Increased membrane conductance, which reduces the slope of the subthreshold current-voltage relationship, can also reduce the slope of the $f-I$ relationship in the presence of voltage fluctuations (Mitchell and Silver, 2003; Prescott and De Koninck, 2003; Silver, 2010). More recent experimental work, however, has indicated that the ability for voltage fluctuations to reduce gain is not broadly applicable to principal cortical neurons (Higgs et al., 2006; Arsiero et al., 2007; Cardin et al., 2008). In addition, voltage fluctuations have also been shown to restore spike firing in cells unable to generate sustained firing (Slee et al., 2005; Prescott et al., 2006; Lundstrom et al., 2009). As a result, a more comprehensive understanding of the interactions between changes in membrane conductance and voltage fluctuations is required. We therefore decided to investigate the effects of changes of membrane conductance and membrane voltage fluctuations on the basic input-output relationship of CA1 pyramidal neurons using dynamic clamp.

Previous work in CA1 pyramidal cells has established that changes in mean membrane conductance, similar to those reported in vivo, alter basic electrophysiological properties. In particular, increased conductance leads to an instantaneous depolarization in spike voltage threshold (Prescott et al., 2006, 2008; Fernandez and White, 2010), which reduces $\mathrm{Na}^{+}$current availability, increases spike frequency adaptation, and decreases gain (Fernandez and White, 2010). Here we show that the introduction of membrane voltage fluctuations under increased membrane conductance restores $\mathrm{Na}^{+}$current and reduces spike frequency adaptation. Contrary to past results, this causes voltage fluctuations to leave output gain unchanged relative to increased membrane conductance alone (Chance et al., 2002; Mitchell and Silver, 2003). 


\section{Materials and Methods}

Tissue preparation. All experimental protocols were approved by the University of Utah Institutional Animal Care and Use Committee. Horizontal sections of hippocampus and entorhinal cortex were prepared from 21- to 31-d-old Long-Evans rats of either sex. All chemicals were obtained from Sigma unless otherwise noted. After anesthetization with isoflurane and decapitation, brains were removed and immersed in $0^{\circ} \mathrm{C}$ solution consisting of the following (in mM): sucrose (215), $\mathrm{NaHCO}_{3}$ (25), D-glucose (20), $\mathrm{KCl}(2.5), \mathrm{CaCl}_{2}(0.5), \mathrm{NaH}_{2} \mathrm{PO}_{4}$ (1.25), and $\mathrm{MgCl}_{2}$ (3), buffered to $\mathrm{pH} 7.4$ with $95 / 5 \% \mathrm{O}_{2} / \mathrm{CO}_{2}$. Horizontal slices were cut to a thickness of $400 \mu \mathrm{m}$ (VT 1200; Leica Microsystems). After the cutting procedure, slices were incubated in artificial CSF (ACSF) at $30^{\circ} \mathrm{C}$ for 20 min before being cooled to room temperature $\left(20^{\circ} \mathrm{C}\right)$. The ACSF consisted of the following (in mM): $\mathrm{NaCl}$ (125), $\mathrm{NaHCO}_{3}$ (25), D-glucose (25), $\mathrm{KCl}(2), \mathrm{CaCl}_{2}$ (2), $\mathrm{NaH}_{2} \mathrm{PO}_{4}$ (1.25), and $\mathrm{MgCl}_{2}$ (1), buffered to $\mathrm{pH}$ 7.4 with $95 / 5 \% \mathrm{O}_{2} / \mathrm{CO}_{2}$. After the incubation period, slices were moved to the stage of an infrared differential interference contrast-equipped microscope (Axioscope 2+; Zeiss). ACSF contained $10 \mu \mathrm{M}$ CNQX, 50 $\mu \mathrm{M}$ picrotoxin, and $30 \mu \mathrm{M} \mathrm{AP}-5$ to block ionotropic synaptic activity. All recordings were conducted between 32 and $34^{\circ} \mathrm{C}$.

Electrophysiology. Electrodes were drawn on a horizontal puller (P97; Sutter Instruments) and filled with an intracellular solution consisting of the following (in mM): K-gluconate (120), $\mathrm{KCl}(20)$, HEPES (10), diTrisPhCr (7), $\mathrm{Na}_{2} \mathrm{ATP}(4), \mathrm{MgCl}_{2}$ (2), Tris-GTP (0.3), EGTA (0.2), buffered to $\mathrm{pH} 7.3$ with $\mathrm{KOH}$. Final electrode resistances were between 3 and 4 $\mathrm{M} \Omega$, with access resistance values between 4 and $12 \mathrm{M} \Omega$. Electrophysiological recordings were performed with a current-clamp amplifier (Axoclamp 2B; Molecular Devices), and data were acquired using custom software developed in Matlab (version 2007b; Mathworks) using the data acquisition toolbox or Clampex (version 10; Molecular Devices). In some experiments, tetrodotoxin (TTX) (5 nM) was bath applied, and recordings were taken after a $10-15$ min incubation period.

For voltage-clamp experiments, electrodes were prepared as described above and filled with intracellular solution consisting of the following (in $\mathrm{mm})$ : Cs-methanesulfonate (100), $\mathrm{NaCl}$ (10), $\mathrm{KCl}$ (1), TEA-Cl (15), EGTA (5.5), HEPES (10), $\mathrm{CaCl}_{2}$ (0.5), $\mathrm{MgCl}_{2}$ (2), diTrisPhCr (7), $\mathrm{Na}_{2} \mathrm{ATP}(4)$, and TrisGTP (0.3), with pH set to $\sim 7.25$ with $\mathrm{CsOH}$. Slices were bathed in extracellular solution consisting of the following (in $\mathrm{mM}$ ): $\mathrm{NaCl}$ (25), $\mathrm{NaHCO}_{3}$ (25), $\mathrm{NaH}_{2} \mathrm{PO}_{4}$ (1.25), $\mathrm{CsCl}$ (5), 4-AP (5), TEA-Cl (15), $\mathrm{MgCl}_{2}$ (3), $\mathrm{CdCl}_{2}(0.2), \mathrm{KCl}(1)$, D-glucose (25), NMDG (75), and $\mathrm{CaCl}_{2}(1)$, set to $\mathrm{pH} 7.4$ with $\mathrm{CsOH}$ and gassed with $95 / 5 \% \mathrm{O}_{2} / \mathrm{CO}_{2}$. Recordings were performed using a Multiclamp 700B amplifier (Molecular Devices) and pClamp 10 (Molecular Devices) software. Access resistance ranged between 4 and $10 \mathrm{M} \Omega$ and access resistance compensation between 10 and $30 \%$ was routinely used. Positive voltage steps elicited transient inward currents, which were completely blocked by $500 \mathrm{~nm}$ TTX and are referred to as $\mathrm{Na}^{+}$currents throughout the remainder of this article. In addition to the reduced $\mathrm{Na}^{+}$driving force, we used low concentrations of TTX (15-20 nM) to obtain current amplitudes suitable for voltage-clamp. All current traces were subjected to off-line subtraction of the linear leak according to Ohm's law. Capacitance artifacts were subtracted by subtracting scaled responses to a voltage step from $-80 \mathrm{mV}(1 \mathrm{~s})$ to $-70 \mathrm{mV}(500 \mathrm{~ms})$. Leak subtraction and capacitance corrections were done using custom routines in Matlab. A calculated liquid junction potential of $10 \mathrm{mV}$ was subtracted from all command voltages.

For dynamic-clamp experiments, the current-clamp amplifier was driven by an analog signal from an $\times 86$ personal computer running Real-Time Application Interface Linux and an updated version of the Real-Time Linux Dynamic Clamp (Dorval et al., 2001) called Real-Time Experimental Interface (Bettencourt et al., 2008). For all experiments, a noisy synaptic stimulus was generated using two independent conductances representing synaptic excitation (AMPA-like) and inhibition $\left(\mathrm{GABA}_{\mathrm{A}}\right.$-like):

$$
I_{\text {noise }}=g_{e}(t)\left(V_{\text {mem }}-E_{e}\right)+g_{i}(t)\left(V_{\text {mem }}-E_{i}\right) .
$$

The reversal potentials for excitation $\left(E_{e}\right)$ and inhibition $\left(E_{i}\right)$ were set to 0 and $-75 \mathrm{mV}$, respectively. As in previous studies (Destexhe et al., 2001;
Fellous et al., 2003), the terms $g_{e}(t)$ and $g_{i}(t)$ were implemented using an Ornstein-Uhlenbeck process and modeled using the following equations:

$$
\frac{d g_{e}}{d t}=\frac{\left(g_{e}-g_{e \mu}\right)}{\tau_{e}}+\sqrt{D_{e}} X_{e}(t), \frac{d g_{i}}{d t}=\frac{\left(g_{i}-g_{i \mu}\right)}{\tau_{i}}+\sqrt{D_{i}} X_{i}(t) .
$$

In the equations, $g_{e \mu}$ and $g_{i \mu}$ represent the mean conductance for excitation and inhibition. $D_{e}$ and $D_{i}$ represent the diffusion coefficients, and $\chi_{e}$ and $\chi_{i}$ are Gaussian white noise terms with unit SD. The terms $\tau_{e}$ and $\tau_{i}$ correspond to the time constant for excitation and inhibition, which were set to 2 and $8 \mathrm{~ms}$, respectively. Unless otherwise noted, we set the $D_{e}$ and $D_{i}$ values so as to generate membrane voltage fluctuations with an SD between 2 and $3 \mathrm{mV}$ for experiments and simulations. For conditions where fluctuations were added without a mean conductance component, we used the Ornstein-Uhlenbeck processes with the mean conductance values set to zero $\left(g_{e \mu}\right.$ and $\left.g_{i \mu}=0\right)$. We refer to cases in which the mean conductance values were set to zero as low conductance with membrane voltage fluctuations. In cases where mean membrane conductance was increased, $g_{e \mu}$ and $g_{i \mu}$ were kept at a 1:4 ratio (e.g., usually set to 2 and $8 \mathrm{nS}$, respectively). Conductance equations were solved numerically using the same method described by Destexhe et al. (2001). The primary reason for using an Ornstein-Uhlenbeck process was that it allowed a reliable control of membrane voltage fluctuation size and mean added conductance since the two terms can be manipulated independently.

In all experiments shown, values for $g_{e}$ and $g_{i}$ were allowed to fall below zero (there was no rectification). This was done to avoid an increase in membrane conductance in a scenario where $g_{e}$ and $g_{i}$ are not allowed to fall below the mean $g_{e \mu}$ and $g_{i \mu}=0$. Thus, in our experimental conditions where mean added conductance was set to zero (low conductance condition), a small positive or negative conductance was introduced at some time points ( $<1 \mathrm{nS}$ for CAl pyramidal cells). This was never an issue in the high-conductance state when $g_{e \mu}$ and $g_{i \mu}$ were much greater than zero. To verify that this did not influence our results, we repeated our experiments using biexponential inhibitory and excitatory synaptic events driven by a Poisson process. Individual synaptic events were modeled as either a conductance event (using the same reversals as before) or as a pure current event. For these experiments, we focused on the effects of mean spiking voltage and spike frequency adaptation using control, current-based Poisson synaptic activity, and conductance-based Poisson synaptic activity. We did not observe any obvious difference from the results presented here ( $n=19$, data not shown). Thus, current-based Poisson activity produced the same results as the Ornstein-Uhlenbeck processes with zero mean conductances.

In cases where conductance was added without membrane voltage fluctuations, a linear leak conductance was introduced via dynamic clamp using the following equation:

$$
I_{\text {leak }}=g_{\text {leak }}\left(V_{\text {mem }}-E_{\text {leak }}\right) \text {. }
$$

For all experiments, $E_{\text {leak }}$ was set to $-75 \mathrm{mV}$. The sample rate of the dynamic clamp was set to $10 \mathrm{kHz}$. A measured junction potential of $\sim 10$ $\mathrm{mV}$ was subtracted from all recordings and taken into account during dynamic-clamp experiments. Data were collected between 5 and $20 \mathrm{kHz}$, and filtered at $3 \mathrm{kHz}$. Gain measurements were taken within the linear range of the $f-I$ relationship (usually below $35 \mathrm{~Hz}$ for initial firing rates and $<15 \mathrm{~Hz}$ for steady-state firing rates).

Anatomical analysis. Identification of interneurons was established using biocytin in the intracellular recording solution and a post hoc analysis using immunofluorescence. Approximately $50 \%$ of CA1 interneuron recordings were done with an intracellular solution containing $0.6 \%$ biocytin (Invitrogen) by weight. After cells were allowed to passively fill with biocytin for approximately $1 \mathrm{~h}$, they were fixed in $4 \%$ paraformaldehyde. To visualize neurons, slices were washed three times for $15 \mathrm{~min}$ in $0.1 \mathrm{M}$ PBS and then incubated for $2-4 \mathrm{~h}$ in a solution containing $1 \mathrm{mg} / \mathrm{mL}$ streptavidin-Alexa 532 (Invitrogen) and $0.75 \mathrm{~mL} / 100 \mathrm{~mL}$ Triton X-100 (Sigma) in PBS. After another three washes in PBS, slices were mounted on microscope slides in Mowiol. Cells were imaged using an Olympus FT1000 confocal microscope. 
Simulations. All simulations were solved in Matlab using a fourth order Runge-Kutta or a forward Euler method with a time step of $0.05 \mathrm{~ms}$. Equations for the model were as follows:

Membrane voltage:

$$
C \frac{d V}{d t}=I_{E}-g_{\mathrm{Na}^{+}} m_{\infty} h^{3}\left(V-E_{\mathrm{Na}^{+}}\right)-g_{\text {leak }}\left(V-E_{\text {leak }}\right),
$$

$\mathrm{Na}^{+}$conductance activation:

$$
m_{\infty}=\frac{1}{1+e^{\left(\left(V-V_{\text {half }}\right) /-k\right)}} V_{\text {half }}=-30, k=4 \text {, }
$$

$\mathrm{Na}^{+}$conductance inactivation:

$$
\begin{gathered}
\frac{d h}{d t}=\frac{h_{\infty}-h}{\tau_{h}}, h_{\infty}=\frac{1}{1+e^{\left(\left(V-V_{\text {half }}\right) /-k\right)}} V_{\text {half }}=-52, k=-2, \\
\tau_{h}=200 \mathrm{~ms}
\end{gathered}
$$

Additional model parameters were as follows: $C=1.5 \mu \mathrm{F} / \mathrm{cm}^{2}$, $g_{\mathrm{Na}^{+}}=6 \mathrm{mS} / \mathrm{cm}^{2}, g_{\text {leak }}=0.03 \mathrm{mS} / \mathrm{cm}^{2}, E_{\mathrm{Na}^{+}}=50 \mathrm{mV}, E_{\text {leak }}=-65 \mathrm{mV}$. $I_{E}$ in the current balance equation refers to driving current. For spike repolarization, membrane voltage was reset to $-65 \mathrm{mV}$ subsequent to crossing 15 $\mathrm{mV}$. The $\mathrm{Na}^{+}$conductance activation $(m)$ was modeled using only the steady-state function. Hence, the activation variable associated with $\mathrm{Na}^{+}$ conductance equilibrates instantaneously with membrane voltage.

Analysis and statistics. All analysis were carried out in Matlab (version 2007b) using custom software and/or built-in functions. Spike times were determined using a threshold crossing for membrane voltage. To measure the $f-I$ relationship and gain, cells were held between -75 and $-70 \mathrm{mV}$ using DC current before eliciting spike firing with a square current step protocol. The frequency of spike firing was calculated from the mean interspike interval (ISI) value. For the initial frequency, the value was calculated from the first three interspike intervals. Steady-state frequency was taken from the mean ISI value for the last third of the $4 \mathrm{~s}$ current pulse used to drive spike firing during $f-I$ measurements. This corresponded to the steady-state region of the spike frequency-versustime relationship. The spike rate of rise was defined as the maximum positive value of the membrane voltage derivative associated with the spike trajectory. Gain was calculated from a linear regression analysis of the linear portion of the $f-I$ relationship and normalized to the condition that produced maximum gain. Unless otherwise noted, mean spiking voltage was defined as the mean voltage value during spike firing. This value includes both the spike and the interspike voltage trajectory. The current protocol for input impedance measurements consisted of a white noise current input with a frequency cutoff of $100 \mathrm{~Hz}$. Impedance $[Z(f)]$ measures (data not shown) were calculated by taking the ratio of the Fourier transform of the membrane voltage response and current input stimulus. Statistical significance was determined using either a one-way or a two-way ANOVA. For repeated measures, statistical difference was determined using Tukey's honestly significant criteria. Means are presented as the SEM.

\section{Results}

Interneurons and pyramidal cells differ in their response to conductance-based voltage fluctuations

To establish the effects of membrane voltage fluctuations and conductance on neuronal output, we started by measuring the $f-I$ relationship and corresponding gain in CA1 pyramidal cells. We also recorded from a subset of interneurons in the CA1 region to compare and contrast our results from pyramidal cells. Anatomical data, in combination with electrophysiological characterization, identified interneurons as putative bistratified cells (Freund and Buzsáki, 1996; Maccaferri et al., 2000). For both cell types, gain was calculated under four different background conditions. These included low conductance (low g, control neuron with no change in mean conductance), low conductance with voltage fluctuations (low $\mathrm{g}+$ fluctuations, approximating synaptic activ- ity with little change in mean conductance; see Materials and Methods), high membrane conductance alone (high g), and high conductance with voltage fluctuations (high $\mathrm{g}+$ fluctuations, approximating synaptic activity associated with a substantial conductance component). Using this approach, we could measure the effects of voltage fluctuations and conductance in isolation and then combine them to assess possible interactions. This is important given recent experimental evidence that different visual stimuli can evoke membrane voltage fluctuations with or without changing mean membrane conductance in cortical neurons (Cardin et al., 2008).

Conductance and membrane voltage fluctuations were introduced via dynamic clamp and constructed using OrnsteinUhlenbeck processes that modeled excitatory and inhibitory synaptic activity (see Materials and Methods). For high membrane conductance, the amount of conductance added was adjusted so as to reduce the final steady-state input resistance in individual pyramidal cells and interneurons by $\sim 50 \%$. This corresponded to a total added conductance range of $10-15 \mathrm{nS}$ for pyramidal cells and $2-5 \mathrm{nS}$ for interneurons. Input resistance in pyramidal cells was reduced from $80.0 \pm 7.1$ to $48.8 \pm 6.0 \mathrm{M} \Omega$, and from $316 \pm 48$ to $162 \pm 22 \mathrm{M} \Omega$ in interneurons.

For each of the four conditions outlined above (low g, low $\mathrm{g}+$ fluctuations, high g, high $\mathrm{g}+$ fluctuations), we measured the resulting $f-I$ relationship and gain for the initial and steady-state spike frequency response in interneurons (Fig. $1 A-C$ ) and pyramidal cells (Fig. $1 D-F$ ). Initial and steady-state gain values were determined using the slopes of a linear fit to the linear part of the $f-I$ relationships. Both interneurons and pyramidal cells showed spike frequency adaptation such that gain values were always significantly lower for the steady-state $f-I$ relationships. Gain values were normalized to the condition showing the maximum gain in individual cells.

In interneurons, both the initial and steady-state gains were significantly modified by the stimulus conditions ( $p=0.0003$ and $p=0.0002$, one-way ANOVA; $n=7$ ). Low conductance with voltage fluctuations or high conductance alone reduced the normalized initial gain values by modest but nonsignificant amounts compared with control $(0.83 \pm 0.06$ and $0.90 \pm 0.04$; difference not significant, Tukey's test; $n=7$ ) (Fig. $1 \mathrm{Bi}, \mathrm{Ci}$ ). Likewise, the steady-state gain values under these stimulus conditions were not significantly different from control $(0.84 \pm 0.06 ; p=0.04$ and $0.88 \pm 0.05$, difference not significant, Tukey's test; $n=7$ ) (Fig. $1 \mathrm{Bii}$, Cii). As expected from previous experimental work (Chance et al., 2002; Mitchell and Silver, 2003), the largest changes in initial and steady-state gains were attained under high conductance with membrane voltage fluctuations $(0.64 \pm 0.05 ; p=$ 0.002 and $0.66 \pm 0.04, p=0.001$, Tukey's test; $n=7$ ) (Fig. 1 Bii,Cii).

Next, we measured the effects of the four stimulus conditions on the initial $f-I$ relationship in pyramidal cells. As with interneurons, the initial gain was significantly altered by the four stimulus conditions ( $p<0.0001$, one-way ANOVA; $n=12)$ (Fig. $1 E i, F i)$. The largest reduction in initial gain was attained under high conductance with voltage fluctuations $(0.60 \pm 0.04 ; p<0.0001$, Tukey's test; $n=12$ ) (Fig. 1 Fi). Low conductance with membrane voltage fluctuations also significantly reduced the initial gain by a small amount $(0.79 \pm 0.05 ; p=0.006$, Tukey's test; $n=$ 12) (Fig. $1 \mathrm{Fi}$ ), while high conductance alone had a nonsignificant impact $(0.84 \pm 0.03$; difference not significant, Tukey's test; $n=$ 12) (Fig. $1 \mathrm{Fi}$ ).

In the case of the steady-state gain response in pyramidal cells, the effects of stimulus conditions differed considerably from the 


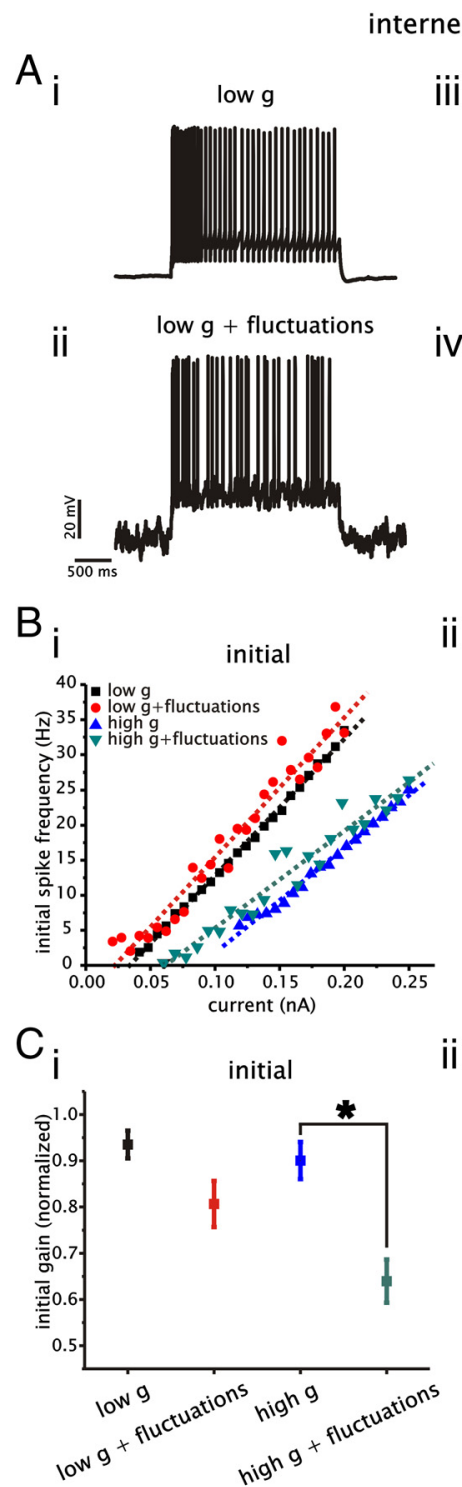

iii

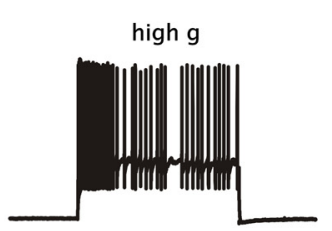

iv

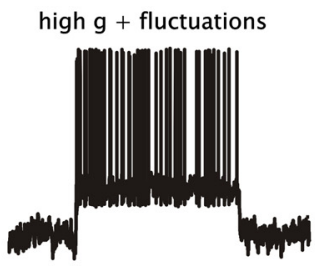

ii

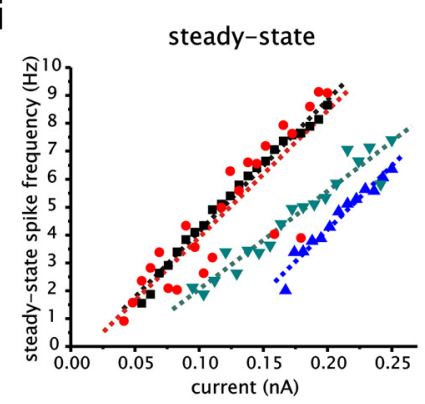

ii

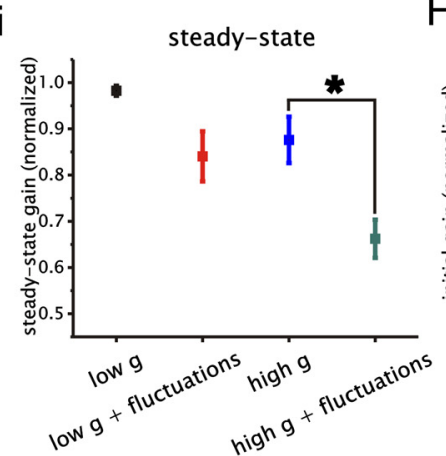

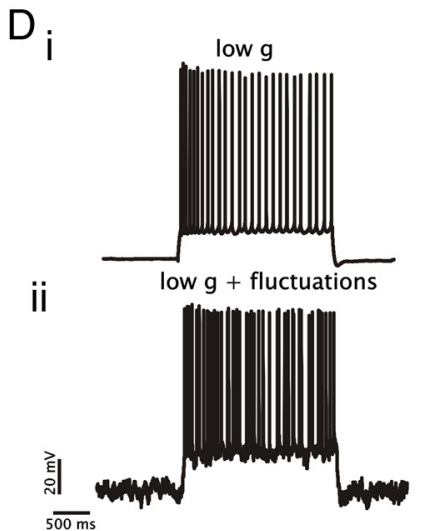

iii

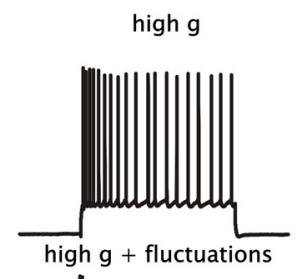

$E_{i}$
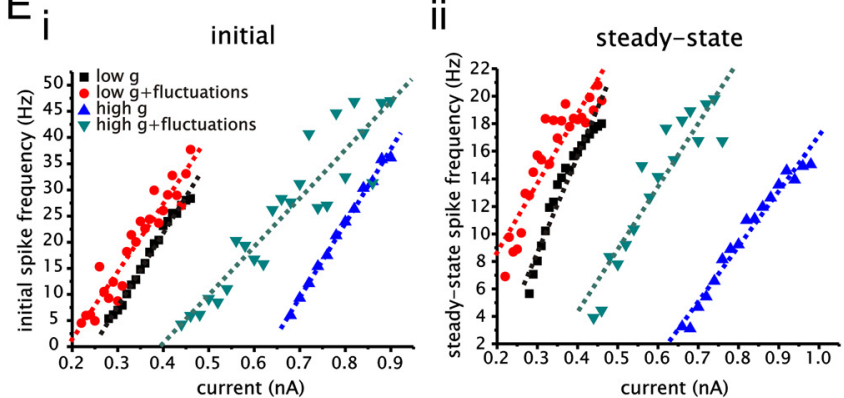

$\mathrm{F}_{\mathrm{i}}$

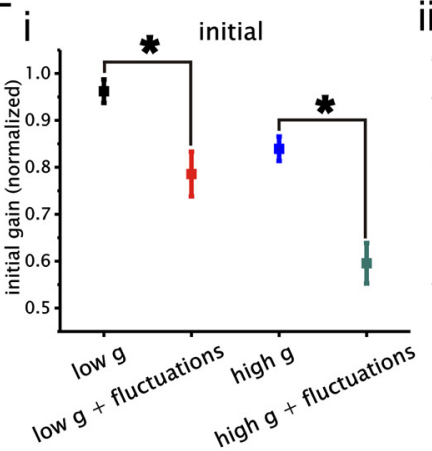

ii

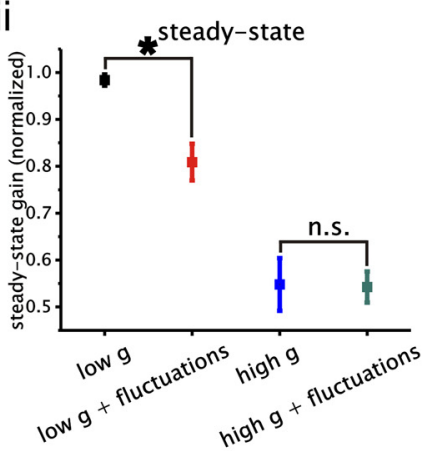

Figure 1. The $f-/$ relationships in CA1 interneurons and pyramidal cells differ in their response to changes in membrane conductance and voltage fluctuations. $A$, Examples of spikes evoked in interneurons by depolarizing current steps under low $\mathrm{g}$ (control conditions) (i), low $\mathrm{g}+$ membrane voltage fluctuations (ii), high $\mathrm{g}$ (iii), and high $\mathrm{g}+$ membrane voltage fluctuations (iv). $\boldsymbol{B}$, Example $f-I$ curves for initial (i) and steady-state (ii) spike frequencies under each of the four stimulus conditions: low $g$ (black), low $g+$ membrane voltage fluctuations (red), high g alone (blue), and high $\mathrm{g}+$ voltage fluctuations (green). Dotted lines represent linear fits to the data points used to calculate the gain (slope) of the $f-/$ relationship. $\boldsymbol{C}$, Plot of normalized gain for initial (i) and steady-state (ii) $f-I$ relationships calculated under each of the four stimulus conditions for CA1 interneurons. The gain was calculated using linear fits to the linear part of the $f-I$ relationship under each condition (dotted lines). Data were normalized to the maximal gain for each neuron. $\boldsymbol{D}$, Examples of spikes evoked in CA1 pyramidal neurons by depolarizing current steps. Same conventions as in $\boldsymbol{A}$ apply. $\boldsymbol{E}$, Example $f-I$ curves for initial (i) and steady-state (ii) spike frequencies under each of the four stimulus conditions. The gain was calculated using linear fits to the linear part of the $f-I$ relationship under each condition (dotted lines). $\boldsymbol{F}$, Plot of normalized gain for initial (i) and steady-state (ii) $f-I$ relationships calculated under each of the four stimulus conditions for $(A 1$ pyramidal cells. Asterisks indicate significant differences $\left({ }^{*} p<0.05\right)$.

cases described above. As before, the gain response was significantly altered by the four stimulus conditions $(p<0.0001$, oneway ANOVA; $n=12$ ) (Fig. 1 Eii,Fii). Low conductance with voltage fluctuations produced a small but significant reduction in steady-state gain $(0.81 \pm 0.04 ; p=0.003$, Tukey's test; $n=12)$. The largest reductions in steady-state gain, however, were attained with either high conductance alone or with high conductance in combination with voltage fluctuations $(0.55 \pm 0.06 ; p<$ 0.0001 and $0.54 \pm 0.03, p<0.0001$, Tukey's test; $n=12$,) (Fig. 1 Fii). Surprisingly, gain measurements for either of these conditions were not significantly different from each other $(p=0.91)$. As a result, under high membrane conductance, adding voltage fluctuations to the stimulus produced no further decrease in gain despite causing a substantial leftward shift in the $f-I$ relationship (Fig. 1 Eii).

We were also interested in the effects of voltage fluctuations in the upper region of the steady-state $f-I$ relationship under high conductance. In particular, we wanted to measure the effect of voltage fluctuations on the maximum steady-state firing rate, given that fluctuations did not change the slope of the linear part of the $f-I$ relationship. To measure the saturation point and maximum steady-state firing frequency, we recorded $f-I$ relationships over a larger current input region than before (Fig. 2). Under high conductance, voltage fluctuations significantly increased the maximum firing frequency when compared with no fluctuations by $5.3 \pm 1.2 \mathrm{~Hz}$ ( $p=0.007$, Student's $t$ test; $n=6)$ (Fig. $2 A, C)$. 
With no increase in mean membrane conductance, the effect of voltage fluctuations on maximum firing rate was significantly smaller ( $p=0.009$, Student's $t$ test) (Fig. $2 C)$, with the value increasing by $1.3 \pm 0.5$ $\mathrm{Hz}$ (Fig. 2B,C). Thus, in a highconductance state, voltage fluctuations provided a pure shift in the linear part of the $f-I$ relationship and an increase in the maximum sustainable firing rate. Conversely, with low conductance, voltage fluctuations led to a reduction in slope and a convergence in the upper region of the $f-I$ relationship with little difference in the maximum firing rate.

The above results indicated that mean spike rate was sensitive to voltage fluctuations at all spike frequencies under high conductance. Unlike the $f-I$ relationship in threshold-linear and simple leaky integrateand-fire models (Tiesinga et al., 2000; Chance et al., 2002; Hansel and van Vreeswijk, 2002; Miller and Troyer, 2002; Mitchell and Silver, 2003), membrane voltage fluctuations can have a purely additive effect on the linear part of the steady-state $f-I$ relationship relative to an increase in membrane conductance alone in CA1 pyramidal cells. Given the unexpected interaction between membrane voltage fluctuations and changes in membrane conductance in pyramidal cells, we followed by investigating the biophysical mechanisms that could account for this result.

\section{Conductance and voltage fluctuations shape spike frequency adaptation in pyramidal cells}

Our previous work in CA1 pyramidal cells established that high conductance alone can reduce the gain of the steady-state $f-I$ relationship (Fig. 1 Fii) through an increase in the magnitude of spike frequency adaptation (Fernandez and White, 2010). As shown in Figures 1 and 2, introduction of membrane voltage fluctuations with increased membrane conductance increases firing rate throughout the steady-state $f-I$ relationship. The increase in firing rate through membrane voltage fluctuations in the lower region of the $f-I$ relationship, near spike threshold, is well established experimentally (Hô and Destexhe, 2000; Chance et al., 2002; Mitchell and Silver, 2003). A potential correlate associated with the increase in firing rate in the upper region of the $f-I$ relationship is that spike frequency adaptation is reduced with voltage fluctuations under high conductance. We hypothesized that the traditional effects of membrane voltage fluctuations near threshold (i.e., increasing spikes at low rates) in conjunction with a reduction of adaptation (i.e., increasing spike rate at higher rates) could produce an additive effect on the $f-I$ relationship by increasing spike rate over a wide region of steady-state spike frequencies and current inputs.

To measure spike frequency adaptation under each of the four stimulus conditions, we drove pyramidal cells with square current steps that evoked similar initial spike rates. An initial rate between 20 and $30 \mathrm{~Hz}$ was chosen as it produced significant adaptation and generated steady-state firing rates corresponding to the upper end of the linear part of the steady-state $f-I$ relationship in pyramidal cells $(\sim 10 \mathrm{~Hz})$. Under low conductance, the time course of spike frequency adaptation over the first 20 interspike intervals did not differ significantly in the presence or absence of membrane voltage fluctuations ( $p=0.46$, two-way ANOVA; $n=12$ ) (Fig. $3 A$ ). Introducing voltage fluctuations under high membrane conductance significantly altered the time course of spike frequency adaptation ( $p<0.0001$, two-way ANOVA; $n=$ 12) (Fig. 3B). We quantified the magnitude of adaptation using an adaptation ratio, which was defined as the initial spike frequency divided by the steady-state value. The adaptation ratio in pyramidal cells was significantly changed by the four stimulus conditions ( $p=0.0005$, one-way ANOVA; $n=12$ ) (Fig. $3 C$ ). Under low conductance, fluctuations had no effect on the adaptation ratio ( $1.89 \pm 0.20$ vs $1.92 \pm 0.12$; difference not significant, Tukey's test; $n=12$ ) (Fig. $3 C$ ). High membrane conductance alone significantly increased the adaptation ratio relative to control (3.27 \pm 0.45 vs $1.92 \pm 0.12 ; p=0.01$, Tukey's test; $n=12$ ) (Fig. 3C). Conversely, high conductance with voltage fluctuations significantly reduced the magnitude of spike frequency adaptation back to levels observed under control conditions (1.95 \pm 0.09; difference not significant, Tukey's test; $n=12$ ) (Fig. 3C). Thus, membrane voltage fluctuations had a differential effect on spike frequency adaptation depending on the magnitude of membrane conductance. We also repeated this analysis in interneurons. Unlike pyramidal cells, the adaptation ratio did not differ as a function of the four stimulus conditions ( $p=0.56$, one-way ANOVA; $n=7$ ) (Fig. 3D). The lack of change in adaptation in interneurons is consistent with the modest effects of high membrane conductance alone on the steady-state gain.

In summary, high membrane conductance with voltage fluctuations preserved the reduction in steady-state gain established with high conductance alone. Under high membrane conductance, voltage fluctuations increased firing rate at both the lower and upper ranges of the $f-I$ relationship, which produced a leftward shift in the linear part of the $f-I$ relationship. Finally, the increase in spike rate in the upper range of the linear part of the $f-I$ relationship with voltage fluctuations correlates with a reduction in the magnitude of spike frequency adaptation.

\section{Membrane conductance and voltage fluctuations modulate $\mathrm{Na}^{+}$current availability}

Spike frequency adaptation in CA1 pyramidal neurons is associated with cumulative $\mathrm{Na}^{+}$current inactivation (Fernandez and White, 2010). We hypothesized that voltage fluctuations could lead to an increase in the $\mathrm{Na}^{+}$current availability and thus decrease spike frequency adaptation under high membrane conductance.

To assess the relationships among membrane conductance, membrane voltage fluctuations, and $\mathrm{Na}^{+}$current availability during spike generation, we measured the spike rate of rise. Although the somatically measured spike rate of rise does not directly reflect the amount of available $\mathrm{Na}^{+}$current at the action 
A
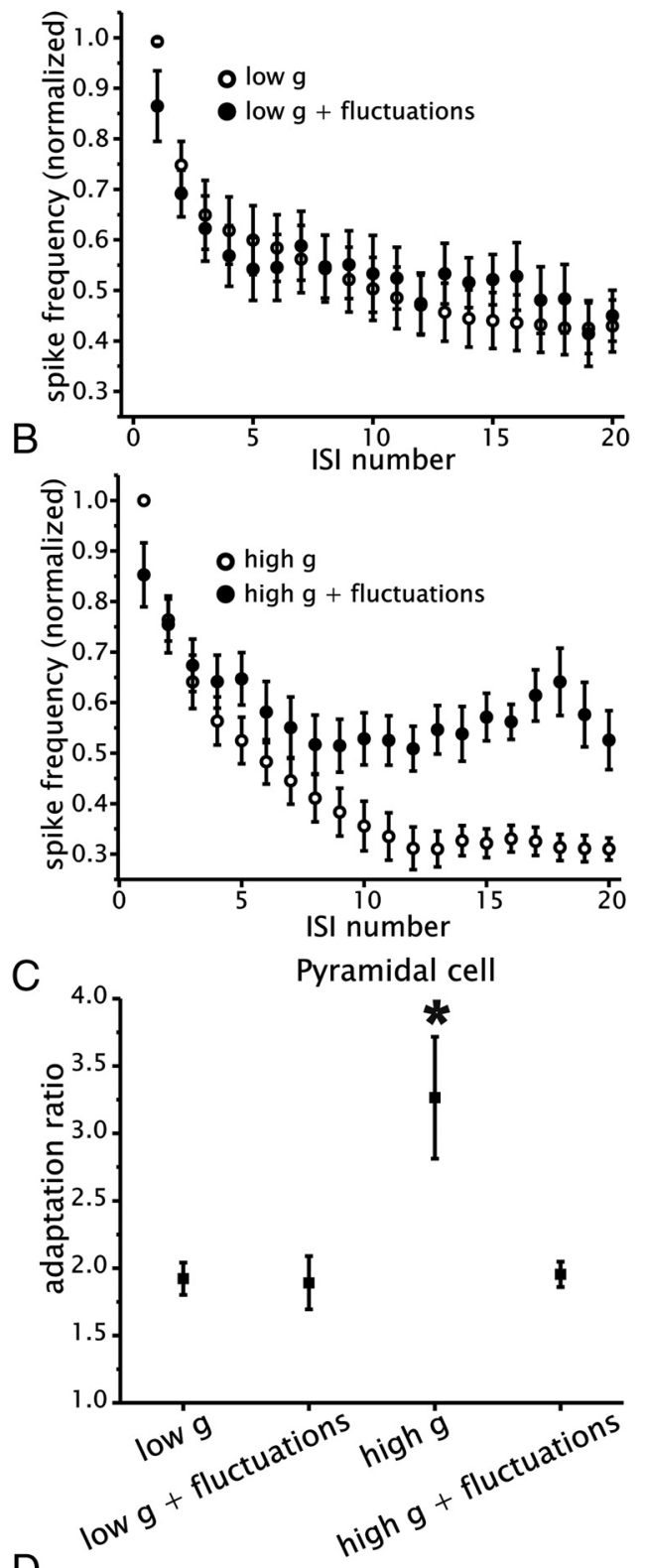

D

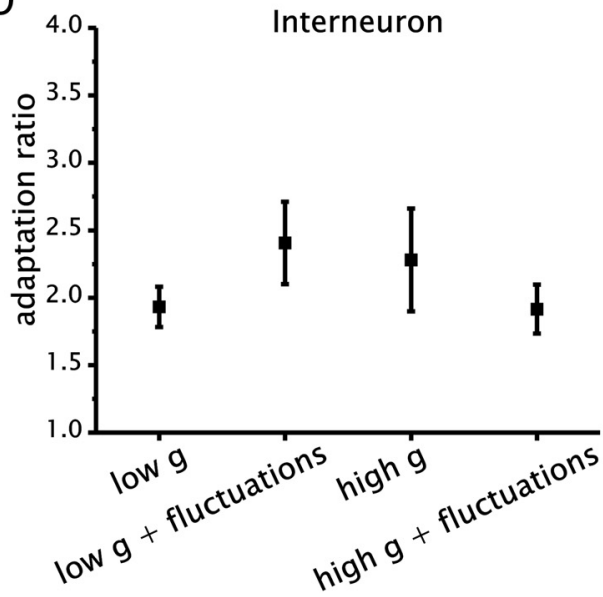

Figure 3. Membrane voltage fluctuations abolish the increase in spike frequency adaptation induced by added conductance. $\boldsymbol{A}$, Averaged normalized spike frequency versus interspike interval number for CA1 pyramidal cells for low conductance (open circles) and low conductance with membrane voltage fluctuations (closed circles). Spike frequency was normalized to the potential initiation site, it provides a good general indication of $\mathrm{Na}^{+}$current availability (Fleidervish et al., 1996; Yau et al., 2010).

We recorded from neurons during steady-state spike firing of a set frequency $(3.39 \pm 0.26 \mathrm{~Hz} ; n=10$; firing frequency was set using DC injection) for $1 \mathrm{~min}$ trials and measured the spike triggered average of membrane voltage associated with changes in conductance and fluctuations. For this series of experiments, we used two different amplitudes of membrane voltage fluctuations ( $\sigma=1 \mathrm{mV}$ or $\sigma=2 \mathrm{mV}$, fluctuation SD was set by manipulating the "diffusion" coefficients of the Ornstein-Uhlenbeck process; see Materials and Methods). The four stimulus conditions had a significant effect on the spike rate of rise ( $p=0.021$, one-way ANOVA; $n=10$ ) (Fig. $4 A, B$ ). Increasing membrane conductance from low to high in the absence of fluctuations reduced the rate of rise from $196 \pm 16$ to $129 \pm 8 \mathrm{mV} / \mathrm{ms}(p=0.0012$, Tukey's test; $n=10$ ) (Fig. $4 B$ ). The combination of high membrane conductance and membrane voltage fluctuations, however, restored the rate of rise to near control values $[167 \pm 15$ $\mathrm{mV} / \mathrm{ms}$ for $\sigma=1 \mathrm{mV}$ vs $180 \pm 18 \mathrm{mV} / \mathrm{ms}$ for $\sigma=2 \mathrm{mV}$, Fig. $4 B$ (only rate of rise for $\sigma=2 \mathrm{mV}$ shown)]. Under low conductance, voltage fluctuations had a modest effect on the rate of rise [202 \pm $14 \mathrm{mV} / \mathrm{ms}$ for $\sigma=1 \mathrm{mV}$ vs $206 \pm 14 \mathrm{mV} / \mathrm{ms}$ for $\sigma=2 \mathrm{mV}$, Fig. $4 B$ (only rate of rise for $\sigma=2 \mathrm{mV}$ shown)].

To confirm that a loss of $\mathrm{Na}^{+}$current occurred throughout all spike frequency measures used to calculate gain, we plotted the mean spike rate of rise as a function of initial and steady-state spike frequency under each of the four stimulus conditions (Fig. $4 C-E)$. A plot of the rate of rise for sequential spikes during steps evoking spike frequency adaptation indicated that increased membrane conductance selectively decreased the steady-state spike rate of rise (Fig. 4Ci). Furthermore, the introduction of voltage fluctuations under high conductance restored the steadystate spike rate of rise to control levels (Fig. 4Cii). To quantify this effect, we segregated data from initial and steady-state spikes and averaged the rates of rise under each of the four stimulus conditions. For initial spikes, the relationship between the spike rate of rise and spike frequency was flat under each of the four stimulus conditions (Fig. 4Di). A measure of the mean spike rate of rise averaged across all initial spike frequencies indicated no significant difference among the four stimulus conditions $(p=0.49$, one-way ANOVA; $n=12$ ) (Fig. $4 \mathrm{Dii}$ ). In contrast, the four stimulus conditions were associated with significantly different rates of rise for steady-state spikes ( $p=0.00032$, one-way ANOVA; $n=12$ ) (Fig. 4 Eii). Increasing membrane conductance from low to high in the absence of voltage fluctuations significantly reduced the mean rate of rise across all steady-state spike frequencies ( $p=0.003$, Tukey's test; $n=12$ ) (Fig. 4 Eii). On the other hand, the introduction of voltage fluctuations under high membrane conductance restored the steady-state spike rate of rise to levels measured under control (difference not significant, Tukey's test; $n=12$ ) (Fig. 4Eii). Finally, voltage fluctuations presented under low membrane conductance had no effect on the steady-state spike rate of rise (difference not significant, Tukey's test) (Fig. 4Eii). Thus, voltage fluctuations restored the $\mathrm{Na}^{+}$current lost with increased membrane conductance alone.

first interspike interval for each neuron. $\boldsymbol{B}$, Averaged normalized spike frequency versus interspike interval number for CA1 pyramidal neurons for high conductance (open circles) and high conductance in combination with membrane voltage fluctuations (closed circles). Same normalization as in $\boldsymbol{A}$. $\boldsymbol{C}, \boldsymbol{D}$, Plot of population averages of the adaptation ratio (initial frequency divided by steady-state frequency) for CA1 pyramidal cells $(\boldsymbol{C})$ and interneurons $(\boldsymbol{D})$. Asterisk indicates significant difference from all other conditions $\left({ }^{*} p<0.05\right)$. 
A

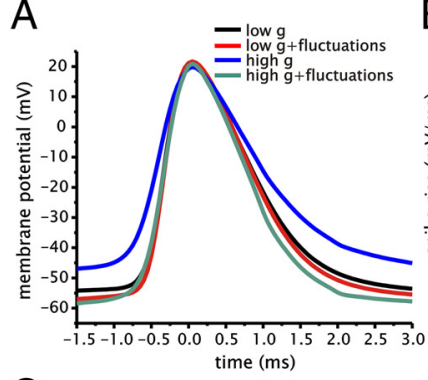

$\mathrm{C}_{\mathrm{i}}$
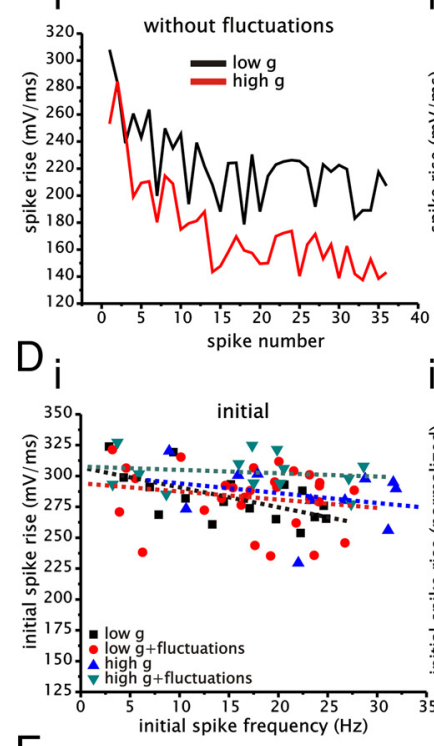

$E_{i}$
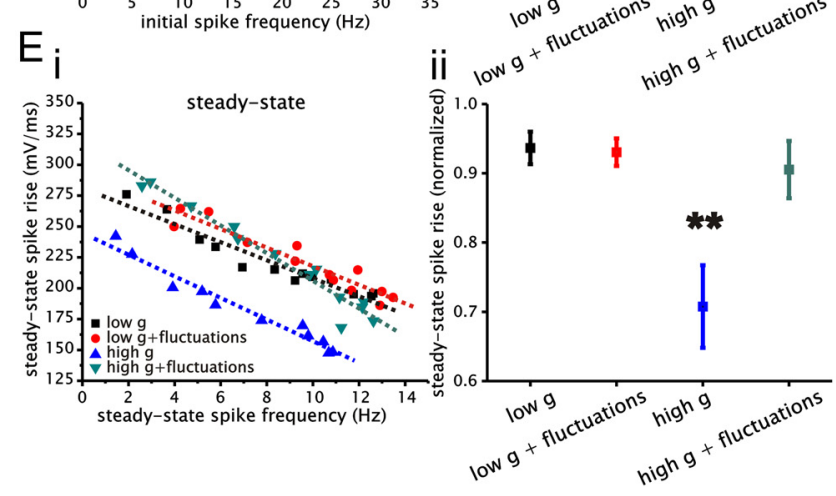

Figure 4. Membrane voltage fluctuations and changes in membrane conductance interact to determine $\mathrm{Na}^{+}$current availability. $\boldsymbol{A}$, Mean spike-triggered averages (4.5 ms window) of the membrane voltage under each of the four stimulus conditions: low conductance (control conditions, black); low conductance with membrane voltage fluctuations (red); high conductance alone (blue); and high membrane conductance with voltage fluctuations (green). Only traces with large membrane voltage fluctuations $(\sigma=2 \mathrm{mV})$ are shown. $\boldsymbol{B}$, Plot of population averages of the maximum spike rate of rise under each of the four stimulus conditions. $\boldsymbol{C}$, Example plots of peak membrane voltage derivatives associated with spiking as a function of spike sequence with (ii) and without (i) membrane voltage fluctuations under low conductance (black) and high conductance (red). D, Plot of the initial spike rates of rise as a function of initial firing frequency under each of the four stimulus conditions. Di, Low conductance (control conditions, black), low conductance with membrane voltage fluctuations (red), with high conductance alone (blue), and high conductance with voltage fluctuations (green). Dotted lines represent linear fits to the data points. Dii, Plot of normalized population data averaged across initial spike frequencies for spike rates of rise under each of the four stimulus conditions. Data were normalized to the maximum rate of rise for each neuron. $\boldsymbol{E}$, Plot of the steady-state spike rates of rise as a function of steady-state firing frequency under each of the four stimulus conditions (i). Eii, Plot of normalized population data averaged across steady-state spike frequencies for spike rates of rise under each of the four stimulus conditions. Samenormalization as in Dii. Asterisks indicate significant difference from all other conditions $\left({ }^{* *} p<0.01\right.$; $\left.{ }^{* * *} p<0.001\right)$.

\section{Membrane conductance and voltage fluctuations determine mean spiking voltage}

We and others have previously shown that increasing membrane conductance alone has a strong depolarizing effect on mean spiking voltage (Prescott et al., 2006; Fernandez and White, 2010). We hypothesized that fluctuations could hyperpolarize mean spiking voltage and lead to an increase in $\mathrm{Na}^{+}$current availability, and thus decrease spike frequency adaptation under high membrane conductance.

We started by measuring the effects of membrane voltage fluctuations on mean spiking voltage under low and high membrane conductance (Fig. 5A). As before, we recorded from neurons during steady-state spike firing of a set frequency for $1 \mathrm{~min}$ trials (same dataset shown in Fig. $4 A, B ; n=10$ ). Construction of spike-triggered membrane voltages revealed a substantial depolarization of mean spiking voltage (mean voltage preceding the spike) under high conductance alone ( $-53.1 \pm 0.7$ vs $60.7 \pm 0.6$ $\mathrm{mV} ; p<0.0001$, one-way ANOVA, Tukey's test; $n=10$ ) (Fig. $5 A, B)$. The combination of high membrane conductance and voltage fluctuations hyperpolarized mean spiking voltage relative to an increase in conductance alone $(-57.9 \pm 0.9 \mathrm{mV}$ for $\sigma=1$ $\mathrm{mV}$ and $-62.6 \pm 1.1 \mathrm{mV}$ for $\sigma=2 \mathrm{mV}$ ) (Fig. 5A,B). Finally, under low conductance, voltage fluctuations had a small effect on mean spiking voltage $(-61.4 \pm 0.7 \mathrm{mV}$ for $\sigma=1 \mathrm{mV}$ and $-63.1 \pm 1.0 \mathrm{mV}$ for $\sigma=2 \mathrm{mV}$ ). We also found that the spike rate of rise correlated strongly with mean spiking voltage (linear fit, $r=-0.93, p=0.008$ ) (Fig. 5C). This suggested that hyperpolarization of mean spiking voltage by voltage fluctuations under high conductance increased $\mathrm{Na}^{+}$current availability.

Next, we tested whether mean spiking voltage was hyperpolarized throughout the entire linear range of the steady-state $f-I$ relationship. To measure this in pyramidal cells, we plotted the steady-state spike frequency-voltage $(f-V)$ relationship under each of the four stimulus conditions (Fig. 5D). For these experiments, we defined mean spiking voltage as the average of the last third of the voltage trace (same region used for the steady-state $f-I$ relation), which was then averaged across spike frequencies for individual cells for statistical comparison (Fig. 5E). We also measured the slope of the $f-V$ relationship to assess whether there were any signs of convergence in the upper range of spike firing frequencies between the different stimulus conditions.

The four stimulus conditions had significantly different mean spiking voltage ranges ( $p<0.0001$, one-way ANOVA; $n=12$,) (Fig. $5 E)$ and $f-V$ slopes $(p<0.0001$, one-way ANOVA; $n=12)$ (Fig. 5F). Under low conductance, the introduction of membrane voltage fluctuations had no significant effect on mean spiking voltage $(-58.8 \pm 0.70$ vs $-59.0 \pm 0.95 \mathrm{mV} ; n=12$; Tukey's test) but significantly reduced the slope of the $f-V$ relationship $(0.95 \pm 0.03$ vs $0.63 \pm 0.03 ; p<0.0001$, Tukey's test; $n=12)$. This led to a convergence in $f-V$ relationships at high spike rates (Fig. 5D). Under high membrane conductance, however, membrane voltage fluctuations had a large and significant effect on mean spiking voltage $(-53.7 \pm 1.1$ vs $-57.7 \pm 0.83 \mathrm{mV} ; p<$ 0.0001 , Tukey's test; $n=12$ ) (Fig. $5 E$ ), but a weaker effect on the slope of the $f-V$ relationship $(0.80 \pm 0.06$ vs $0.65 \pm 0.06$; nonsignificant difference, Tukey's test; $n=12$ ) (Fig. $5 F$ ). Like the steady-state $f-I$ relationship, the $f-V$ relationship was largely shifted with weak signs of convergence in the upper spike frequency range (Fig. 5D). This indicated that voltage fluctuations decreased mean spiking voltage over the entire linear range of the $f-V$ and $f-I$ relationships. We hypothesized that the hyperpolarization was due to the combination of a fluctuation-induced spike generation below the spike voltage threshold at low firing 
A

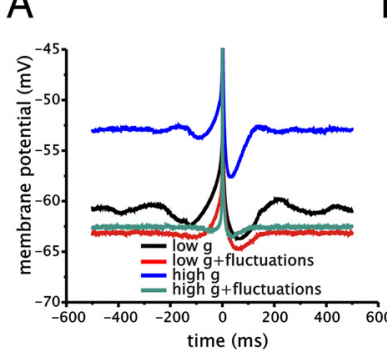

D

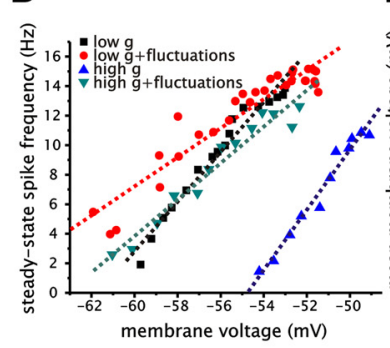

$E$

B
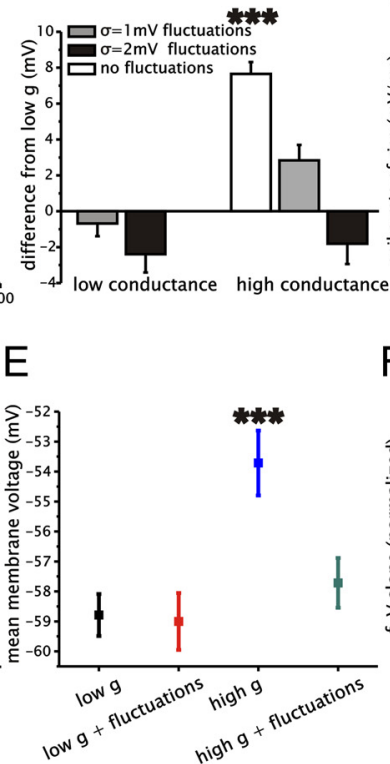

C

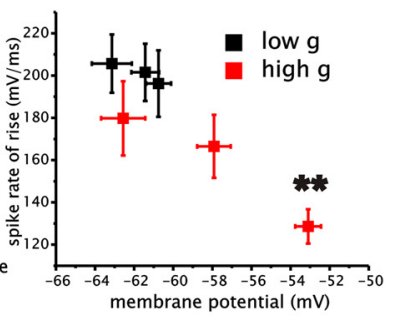

F

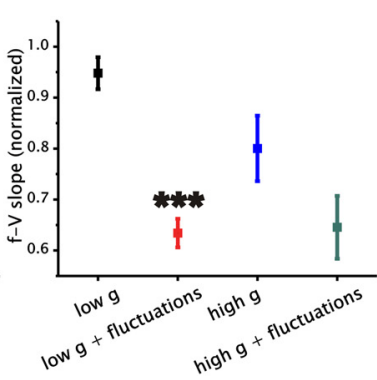

Figure 5. Membrane voltage fluctuations and changes in membrane conductance interact to determine mean spiking voltage. A, Mean spike-triggered averages (1000 ms window) of the membrane voltage under each of the four stimulus conditions: low conductance (control conditions, black); low conductance with membrane voltage fluctuations (red); with high conductance alone (blue); and high membrane conductance with voltage fluctuations (green). Only traces with large membrane voltage fluctuations $(\sigma=2 \mathrm{mV})$ are shown. $B$, Plot of average differences in mean spiking voltage between low and high conductance with or without membrane voltage fluctuations. C, Plot of average maximum spike rate of rise as a function of mean spiking voltage for low conductance (black) and high conductance (red) under three different amplitudes of membrane voltage fluctuations (control, $\sigma=$ $1 \mathrm{mV}$, and $\sigma=2 \mathrm{mV}$ ). For both low conductance (black) and high conductance (red), the left, middle, and right points correspond to $\sigma=2 \mathrm{mV}, \sigma=1 \mathrm{mV}$, and control, respectively. $\boldsymbol{D}$, Example plot of steady-state spike frequency as a function of mean membrane voltage ( $f-\eta$ ) under each of the four stimulus conditions. Dotted lines represent linear fits to the data points. $\boldsymbol{E}$, Plot of population averages of the mean membrane voltage across frequencies under each of the four stimulus conditions. $\boldsymbol{F}$, Plot of population averages of the normalized slopes of the steady-state spike frequency-voltage relationships. Data were normalized to the maximum slope for each neuron. $\boldsymbol{E}$, Asterisks indicate significant difference from all other conditions ( $\left.{ }^{* * *} p<0.05\right)$. In all other panels, asterisks indicate significant difference from low conductance without fluctuations. ${ }^{* *} p<0.01 ;{ }^{* * *} p<0.001$.

0.0009, Tukey's test; $n=8)$ gain values significantly, which confirmed the importance of $\mathrm{Na}^{+}$current availability in establishing gain in CA1 pyramidal cells. The introduction of voltage fluctuations with TTX significantly reduced the initial gain compared with TTX alone $(0.33 \pm 0.04$ vs $0.57 \pm 0.08 ; p=0.008$, one-way ANOVA; $n=8$ ) (Fig. $6 B i$ ). In the presence of TTX, steady-state gain, however, was unaffected by membrane voltage fluctuations relative to TTX application alone $(0.48 \pm$ 0.07 vs $0.43 \pm 0.09$, difference not significant, Tukey's test; $n=8$ ) (Fig. 6 Bii). Instead, fluctuations led to a leftward shift in the linear part of the $f-I$ relationship by increasing the firing rate at all current inputs. Consequently, a reduction of $\mathrm{Na}^{+}$ current with TTX produced a differential modulation of initial and steady-state gains by voltage fluctuations in the same manner as high membrane conductance (compare Figs. 6Bii, 1F).

As with high membrane conductance, we hypothesized that the ability of voltage fluctuations to preserve the gain of the steady-state response under TTX was associated with an increase in the availability of $\mathrm{Na}^{+}$current. To measure this, we constructed spike-triggered averages of the membrane voltage under each of the four stimulus conditions (Fig. 6C). The analysis revealed that membrane fluctuations significantly increased the mean spike rate of rise when compared with TTX application alone $(69.4 \pm 8.9$ vs

rates (Hô and Destexhe, 2000; Chance et al., 2002; Mitchell and Silver, 2003; Prescott and De Koninck, 2003) and another, as yet unidentified, mechanism acting at medium to high firing rates. This second mechanism would be related to the ability for voltage fluctuations to increase the maximum firing rate and reduce spike frequency adaptation under high conductance.

\section{Pharmacological and voltage-clamp data provide evidence for the role \\ of $\mathrm{Na}^{+}$current inactivation in determining the gain modulation in CA1 pyramidal cells}

If increases in mean membrane conductance lead to a reduction of $\mathrm{Na}^{+}$current availability, we predicted that a pharmacological reduction of $\mathrm{Na}^{+}$current should qualitatively replicate the effects on steady-state gain observed with increased membrane conductance. Testing this prediction required a reduction of the total available $\mathrm{Na}^{+}$current without blocking the ability for pyramidal cells to generate spikes. For this reason, we used a small concentration of TTX (5 $\mathrm{nM}$ ), which visibly altered spike shapes but did not inhibit spike generation at steady state.

We measured the $f-I$ relationship of pyramidal cells with or without membrane voltage fluctuations in the presence of $5 \mathrm{nM}$ bath-applied TTX. In the absence of voltage fluctuations, bathapplied TTX had a significant impact on both the initial and steady-state gain response ( $p<0.0001$, one-way ANOVA; $n=8$ ) (Fig. 6A,B); TTX reduced both the initial $(0.57 \pm 0.08 ; p=$ 0.0002 , Tukey's test; $n=8)$ and steady-state $(0.43 \pm 0.09 ; p=$
$31.4 \pm 4.0 \mathrm{mV} / \mathrm{ms} ; p<0.0001$, paired Student's $t$ test; $n=16$ ) (Fig. 6D). In general, the spike waveform changes caused by voltage fluctuations were consistent with a greater availability of $\mathrm{Na}^{+}$ current.

A necessary prerequisite for a role of $\mathrm{Na}^{+}$current in influencing pyramidal cell response in the presence of increased membrane conductance and voltage fluctuations is that the voltage dependence of $\mathrm{Na}^{+}$current inactivation lies within the range of observed changes in mean spiking voltage (range, -60 to -40 $\mathrm{mV}$ ). To address this, we measured the voltage dependence of steady-state inactivation of $\mathrm{Na}^{+}$currents in pyramidal neurons. To obtain small $\mathrm{Na}^{+}$current amplitudes amenable to voltageclamp, we used an extracellular solution that generated a reduced $\mathrm{Na}^{+}$current driving force and applied low concentrations of TTX (15-20 nM). Neurons were stepped to a test voltage of -20 $\mathrm{mV}(500 \mathrm{~ms})$ from increasingly positive conditioning voltages starting from $-90 \mathrm{mV}$ ( $1 \mathrm{~s}, 5 \mathrm{mV}$ increment), eliciting transient $\mathrm{Na}^{+}$currents (Fig. 6E). Boltzmann fits to peak $\mathrm{Na}^{+}$current amplitudes for each conditioning voltage revealed a mean halfmaximal level of inactivation at a conditioning voltage of $-52.6 \pm 1.6 \mathrm{mV}(n=4)$ and an 80 to $20 \%$ availability range between $-62.8 \pm 2.1$ and $-42.4 \pm 1.6 \mathrm{mV}$ (Fig. $6 F$ ). These results indicate that there is a steep drop in $\mathrm{Na}^{+}$current availability in the range corresponding to changes in mean spiking voltage induced by increased membrane conductance and fluctuations $(-63$ to $-53 \mathrm{mV})$. These results are also consistent with 


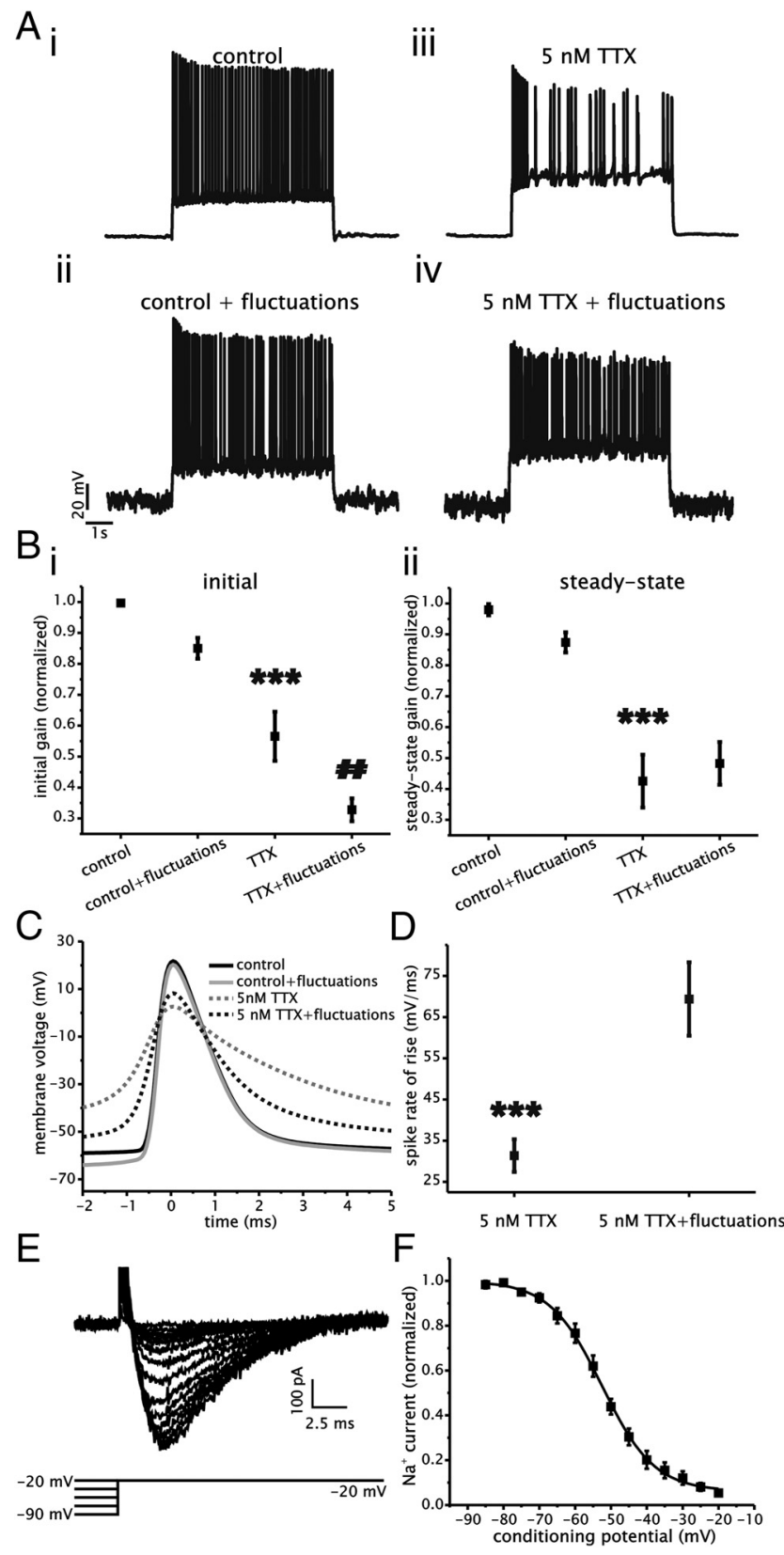

Figure 6. Pharmacological and voltage-clamp data provide evidence for the role of $\mathrm{Na}^{+}$ current inactivation in determining the changes in gain and spike frequency adaptation with increased membrane conductance and voltage fluctuations. $A$, Examples of spikes evoked by depolarizing current steps under low conductance (control conditions (i), low conductance with membrane voltage fluctuations (ii), low conductance with bath applied TTX (iii), and low conductance with membrane voltage fluctuations in the presence of TTX (iv). B, Plot of normalized gain for initial (i) and steady-state (ii) $f-/$ relationships calculated under each of the four stimulus conditions. The gain was calculated using linear fits to the $f-l$ relationship under each condition. Data were normalized to the maximum slope for each neuron. C, Mean spiketriggered averages ( $7 \mathrm{~ms}$ window) of the membrane voltage under low conductance (control conditions, black), low conductance with membrane voltage fluctuations (gray line), low conductance with bath applied TTX (gray dashed line), and low conductance with membrane voltage fluctuations in the presence of TTX (black dashed line). D, Plot of average steady-state spike rates of rise with TTX and TTX with membrane voltage fluctuations under low conductance. $\boldsymbol{E}$, Example $\mathrm{Na}^{+}$current traces recorded by stepping the command voltage to $-20 \mathrm{mV}$ from increasingly positive conditioning voltages ( -90 to $-20 \mathrm{mV}, 5 \mathrm{mV}$ increment). $\boldsymbol{F}$, Plot of average voltage dependence of $\mathrm{Na}^{+}$current inactivation. Black line represents a Boltzmann fit to the data points. Data were normalized to the maximum current amplitude for each neuron. Asterisks indicate significant difference from control: ${ }^{* * *} p<0.001$. Hash marks indicate significant difference from TTX: $p<0.05$. previous voltage-clamp studies of $\mathrm{Na}^{+}$current inactivation in CA1 pyramidal cells (Jung et al., 1997; Mickus et al., 1999).

Inhibitory membrane voltage fluctuations can be excitatory with increased membrane conductance

Surprisingly, our results indicated that hyperpolarization of mean spiking voltage by fluctuations under high membrane conductance increased firing rate (Fig. 5D). This indicates that a net hyperpolarization of mean voltage induced by inhibitory fluctuations could be excitatory under high membrane conductance. This may have important implications for the integration of inhibitory inputs under different membrane conductance states. We followed by testing whether purely inhibitory inputs containing only the $\mathrm{GABA}_{\mathrm{A}}$-like synapse model (reversal potential of $-75 \mathrm{mV}$ ) in our Ornstein-Uhlenbeck process could increase steady-state spike firing rate under high membrane conductance. For these experiments, we compared the pyramidal cell spike output rate using an Ornstein-Uhlenbeck model incorporating both the $\mathrm{AMPA}+\mathrm{GABA}_{\mathrm{A}}$-like processes (same as all previous experiments) or using only the $\mathrm{GABA}_{\mathrm{A}}$-like process. Changes in firing rate were quantified as the difference in spike rate between conditions with and without voltage fluctuations. We recorded from pyramidal cells at steady-state spike frequencies of $\sim 5-10$ $\mathrm{Hz}$ and used identical DC values for both AMPA $+\mathrm{GABA}_{\mathrm{A}}$-like and $\mathrm{GABA}_{\mathrm{A}}$-like inputs.

The use of $\mathrm{AMPA}+\mathrm{GABA}_{\mathrm{A}}$-like fluctuations generated no significant change in initial firing rate under both high and low membrane conductance ( $p>0.45$, one-way ANOVA; data not shown; $n=10$ ). Presenting only $\mathrm{GABA}_{\mathrm{A}}$-like fluctuations produced a modest decrease in initial firing rates under both conditions, although this effect failed to reach significance in the low conductance condition when compared with control (low conductance: $p=0.17$; high conductance: $p=0.017$, one-way ANOVA; $n=10$; data not shown).

In the case of the steady-state spike frequency, we observed a different behavior. The introduction of AMPA $+\mathrm{GABA}_{\mathrm{A}}$-like fluctuations under low membrane conductance increased firing rates by modest amounts ( $p<0.0001$, one-way ANOVA; $n=10)$ (Fig. $7 B$ ). Confirming previous results, AMPA+GABA $A_{A}$ like fluctuations strongly increased spike firing rate under high membrane conductance ( $p<0.0001$, one-way ANOVA; $n=10)$ (Fig. $7 B$ ). In the case of $\mathrm{GABA}_{\mathrm{A}}$-like fluctuations presented under low membrane conductance, mean firing rate was slightly decreased $(p=0.07$, one-way ANOVA; $n=10)$ (Fig. $7 C)$. Despite hyperpolarizing mean membrane voltage, $\mathrm{GABA}_{\mathrm{A}}$-like fluctuations presented under high membrane conductance significantly increased steady-state spike firing compared with high membrane conductance without any voltage fluctuations $(7.6 \pm 0.8$ vs $11.0 \pm 0.9 \mathrm{~Hz} ; p=0.017$, one-way ANOVA; $n=10)($ Fig. $7 A, C)$. Thus, hyperpolarization of mean spiking voltage caused by $\mathrm{GABA}_{\mathrm{A}}$-like synaptic activity can effectively increase steady-state firing rate. This suggests that the inhibitory action of $\mathrm{GABA}_{\mathrm{A}}$ receptor activation will depend on the conductance state of the neuron. Under conditions where background synaptic activity significantly increases membrane conductance, the presence of hyperpolarizing asynchronous inhibitory synaptic activity can be excitatory.

A separation of timescales accounts for the ability of fluctuations to hyperpolarize mean spiking voltage at high spike rate frequencies

To better understand how membrane voltage fluctuations associated with an increase in membrane conductance can hyperpo- 
$A_{i}$

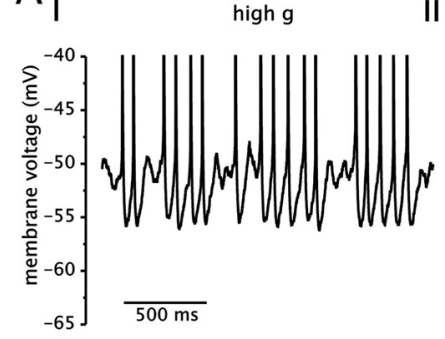

B

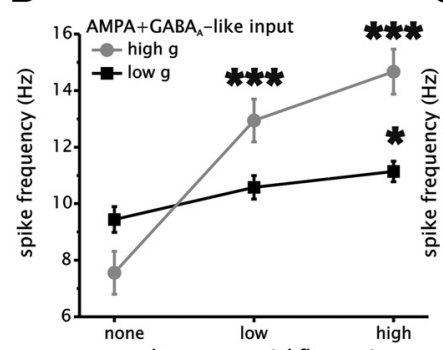

membrane potential fluctuations ii

high $\mathrm{g}+\mathrm{CABA}_{\Lambda}$-like input

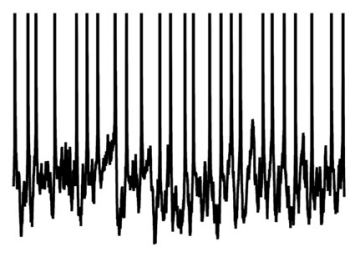

C

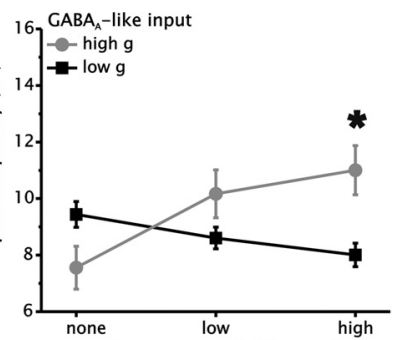

membrane potential fluctuation

Figure 7. $G A B A_{A}$-like inputs increase steady-state firing frequency under high membrane conductance in CA1 pyramidal neurons. $A$, Example steady-state membrane voltage traces under high conductance $(\boldsymbol{i})$, and high conductance in combination with hyperpolarizing membrane voltage fluctuations induced by $G A B A_{A}$-like inputs (ii). Note the increased firing rate at more hyperpolarized membrane voltages. $\boldsymbol{B}$, Steady-state firing frequency versus membrane voltage fluctuation magnitude (none, $\sigma=1 \mathrm{mV}$, and $\sigma=2 \mathrm{mV}$ ) induced by standard $A M P A+G A B A_{A}$-like input. High conductance is shown in gray, low conductance in black. $C$, Spike firing frequency versus membrane voltage fluctuation magnitude induced by $G A B A_{A}$-like input. Note the inverse effect of $\mathrm{GABA}_{A}$-like inputs on steady-state firing frequency under high and low conductance. Same conventions as in Capply. Asterisks indicate significant difference from recordings without membrane voltage fluctuations (either low or high conductance). ${ }^{*} p<0.05 ;{ }^{* * *} p<0.001$.

larize mean spiking voltage and restore $\mathrm{Na}^{+}$current, we used a previously published two-variable conductance-based model of CA1 pyramidal cells that reproduces the reduction in gain and increase in spike frequency adaptation with increased membrane conductance alone (Fernandez and White, 2010). In particular, we were interested in understanding how fluctuations hyperpolarize mean spiking voltage and increase firing rate in the upper region (5-10 $\mathrm{Hz}$ range) of the steady-state $f-I$ relationship, given that this result cannot be accounted for by threshold-linear and leaky integrate-and-fire models (Tiesinga et al., 2000; Chance et al., 2002; Hansel and van Vreeswijk, 2002; Miller and Troyer, 2002; Mitchell and Silver, 2003).

We drove the model under the four stimulus conditions used experimentally. Membrane voltage fluctuations were generated by the same Ornstein-Uhlenbeck process used before. The model qualitatively reproduces the effects of voltage fluctuations under low and high membrane conductance (Fig. 8A). With low conductance $\left(g_{e \mu}+g_{i \mu}=0 \mathrm{mS} / \mathrm{cm}^{2}\right)$, membrane voltage fluctuations induce a small decrease in gain (62 vs $44 \mathrm{~Hz} \cdot \mathrm{cm}^{2} / \mu \mathrm{A}$ ) (Fig. $8 A)$. Conversely, high membrane conductance $\left(g_{e \mu}+g_{i \mu}=0.1\right.$ $\mathrm{mS} / \mathrm{cm}^{2}$ ) alone produces a large reduction in gain that is preserved with the introduction of membrane voltage fluctuations (23 vs $22 \mathrm{~Hz} \cdot \mathrm{cm}^{2} / \mu \mathrm{A}$ ) (Fig. $8 \mathrm{~A}$ ). As in pyramidal cells, increasing membrane conductance leads to a depolarization of the average spike-triggered voltage associated with the upper region of the $f-I$ relationship $(\sim 10 \mathrm{~Hz})$. The combination of increased membrane conductance and voltage fluctuations leads to a hyperpolarization of the average spike-triggered voltage (Fig. $8 \mathrm{~B}$ ). As a consequence of the hyperpolarization, the availability of

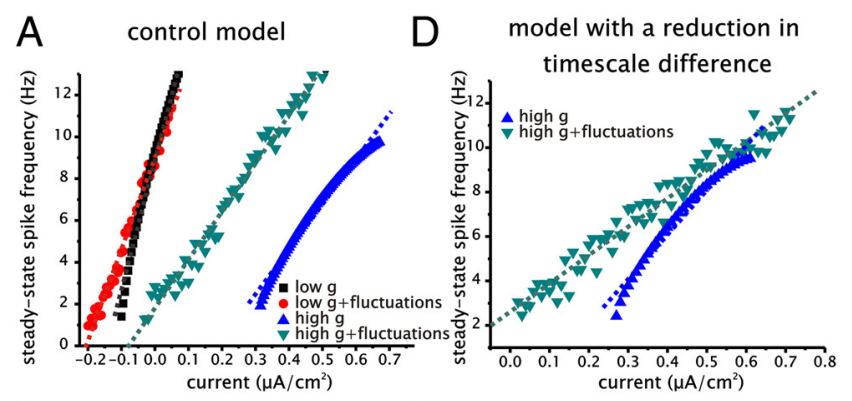

B
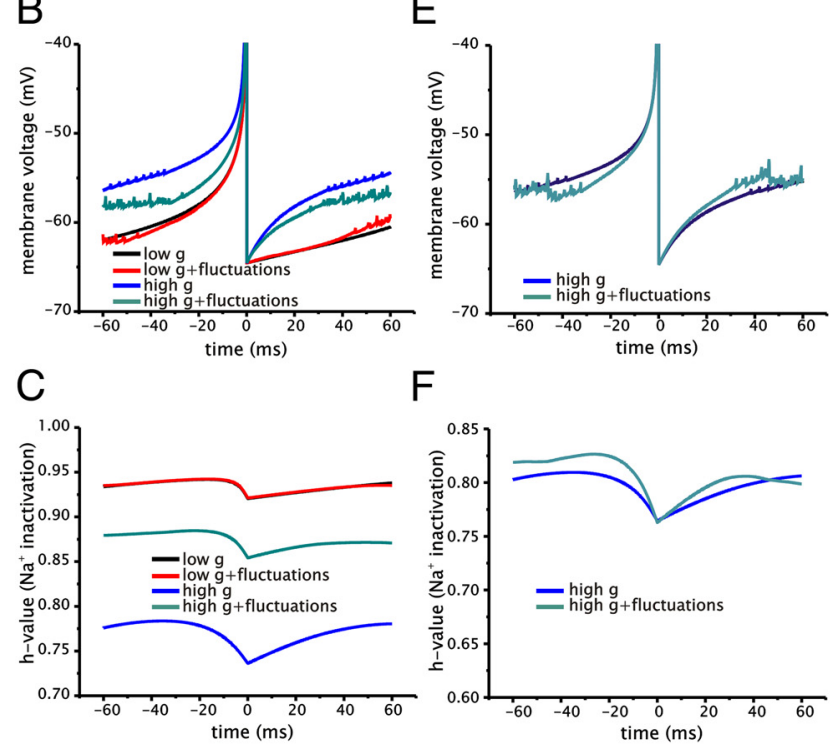

$F$

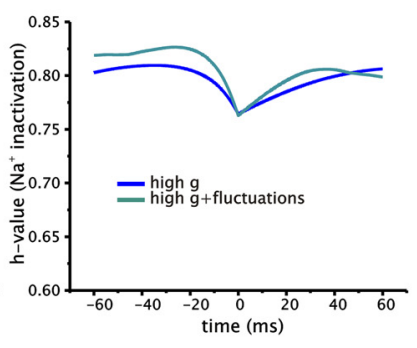

Figure 8. A conductance-based model using a separation of timescale between membrane voltage fluctuations and a slow dampening variable ( $h$ inactivation) is required to reproduce the effects of membrane voltage fluctuations and conductance on the spike firing output in CA1 pyramidal cells. $\boldsymbol{A}$, Frequency-current relationship for the model under four conditions: low conductance (control conditions, black), low conductance with membrane voltage fluctuations (red), high conductance only (blue), and high conductance in combination with membrane voltage fluctuations (green). $\boldsymbol{B}, \boldsymbol{C}$, Mean spike-triggered voltage $(\boldsymbol{B})$ and $h$-variable $(\boldsymbol{C})$ averages under each of the four stimulus conditions. Averages are taken from the model firing at $\sim 10 \mathrm{~Hz}$. $D$, Frequency-current relationship for the model with a reduction in the separation of timescales between the voltage fluctuations and the $h$ variable under each of the four stimulus conditions. $\boldsymbol{E}, \boldsymbol{F}$, Mean spike-triggered voltage $(\boldsymbol{E})$ and $h$-variable $(\boldsymbol{F})$ averages with a reduction in the separation of timescales between the voltage fluctuations and the $h$ variable under added conductance alone (blue) and with added conductance in combination with membrane voltage fluctuations (green). Averages are taken from model firing at $\sim 10 \mathrm{~Hz}$.

$\mathrm{Na}^{+}$current associated with the average spike voltage increases (larger mean $h$-variable values) (Fig. $8 C$ ). In contrast, with low mean membrane conductance, voltage fluctuations have no effect on the average spike-triggered voltage or $\mathrm{Na}^{+}$current availability (Fig. $8 B, C$ ). We should note, however, that the model reproduces the effects of increased conductance and voltage fluctuations on gain using a much steeper steady-state $\mathrm{Na}^{+}$inactivation curve than that observed in CA1 pyramidal cells (Fig. $6 F$ ). This is required because the model generates a smaller change in mean spiking voltage in response to increased membrane conductance relative to CA1 pyramidal cells (compare Figs. 5A, $8 B$ ) and hence requires a steeper drop in $\mathrm{Na}^{+}$current with depolarization. Despite this, we feel the model still provides a useful tool to help explain the experimental results.

Previous modeling work has analyzed the effects of fast membrane voltage fluctuations in conductance-based models with slow dampening variables, such as those represented by the $h$ variable in the above model (Lundstrom et al., 2009). Fast voltage 
fluctuations permit variables associated with the spike upstroke to generate a spike and escape the inhibitory effects of slow dampening variables that cannot equilibrate with fast changes in membrane voltage. Voltage fluctuations have fast frequency components due to the relative short time constants underlying synaptic activity $(<10 \mathrm{~ms})$ and the membrane charge time $(<25$ $\mathrm{ms})$. On the other hand, the process of spike frequency adaptation occurs over hundreds of milliseconds in CA1 pyramidal cells due to the slower dynamics of cumulative $\mathrm{Na}^{+}$current inactivation (Fleidervish et al., 1996; Jung et al., 1997; Mickus et al., 1999). Importantly, a separation of timescales can increase firing rate in the upper range of an $f-I$ relationship since it does not depend on voltage fluctuations generating spikes via a transient excursion past spike threshold.

A key prediction of this mechanism is that a reduction in the separation of timescales between fluctuations and the slow dampening variable should eliminate the ability for membrane voltage fluctuations to increase firing rate and hyperpolarize mean spiking voltage in the upper region of the $f-I$ relationship. To test this, we reduced the time constant of the $h$ variable (from 200 to $120 \mathrm{~ms}$ ), and increased the time constants associated with the Ornstein-Uhlenbeck processes that modeled excitatory and inhibitory activity (both were set to $200 \mathrm{~ms}$ ). With a reduction in the separation of timescales, membrane voltage fluctuations result in a substantial reduction in gain of the $f-I$ relationship under high conductance $\left(22 \mathrm{vs} 13 \mathrm{~Hz} \cdot \mathrm{cm}^{2} / \mu \mathrm{A}\right)($ Fig. $8 D)$. Under these conditions, a measure of the average spike-triggered voltage and associated $h$-variable values in the upper region of the $f-I$ relationship $(\sim 10 \mathrm{~Hz})$ indicates no substantial difference in these variables in the absence or presence of voltage fluctuations (Fig. $8 E, F)$. Thus, reducing the separation of timescales between membrane voltage fluctuations and an intrinsic and slow recovery variable alters the model so that changes in the $f-I$ relationship are more consistent with threshold-linear and leaky integrateand-fire models (Tiesinga et al., 2000; Chance et al., 2002; Hansel and van Vreeswijk, 2002; Miller and Troyer, 2002; Mitchell and Silver, 2003).

In summary, two separate mechanisms help increase firing rate with voltage fluctuations over the linear portion of the $f-I$ relationship. The first mechanism is well established and relates to the ability for voltage fluctuations to elicit spikes through transient excursions past spike threshold. This effect is related to previous studies showing a divisive effect of voltage fluctuations on gain and can be reproduced using an integrate-and-fire model (Chance et al., 2002; Mitchell and Silver, 2003). The second mechanism (separation of timescales) also hyperpolarizes mean spiking voltage (for a given spike frequency rate), but is the only available mechanism in the upper region of the $f-I$ relationship for increasing spike rate since it does not depend on voltage fluctuations crossing the spike voltage threshold. The second mechanism works by having fast voltage fluctuations increase spike rate without recruiting the slow dampening effect of spike frequency adaptation. Under control conditions, positive and negative voltage fluctuations have no net effect on mean spike firing rate in the upper region of the $f-I$ relationship since the positive fluctuations add spikes and negative fluctuations subtract spikes. With high conductance and increased spike frequency adaptation, however, the second mechanism hyperpolarizes mean spiking voltage at higher spike rates through the presence of fast voltage fluctuations that permit spiking without substantial recruitment of spike frequency adaptation.

\section{Fast membrane voltage fluctuations are best suited for increasing firing rate}

The results from the model analysis indicated that the ability for membrane voltage fluctuations to hyperpolarize mean spiking voltage in the upper range of the $f-I$ relationship was attributable to the higher frequency components of the voltage fluctuations. We hypothesized that an optimal fluctuation frequency range exists that would generate a maximum increase in firing rate and restoration of spike shape parameters consistent with an increase in $\mathrm{Na}^{+}$current availability. This frequency range would correspond to changes in membrane voltage that outpace the slow process involved in spike frequency adaptation, but are not significantly filtered by the membrane time constant. To test this, we designed a protocol that contained a sinusoidal current stimulus of different frequencies. The stimulus consisted of an initial $10 \mathrm{~s}$ component with a sinusoid of a set frequency, which was followed by a second component containing only the DC drive (mean during sinusoid) (Fig. 9A). We used a range of frequencies between 1 and $500 \mathrm{~Hz}$ for the sinusoidal component and had pyramidal cells fire at $\sim 10 \mathrm{~Hz}$ under DC drive. The stimulus was applied to cells under control and with increased membrane conductance (using a linear "leak"; see Materials and Methods). The amplitude of the current stimulus was adjusted to compensate for changes in steady-state input resistance caused by the linear leak conductance. Thus, we approximately doubled the amplitude of the sinusoidal stimulus to compensate for the changes in steadystate input resistance. Using this stimulus, we could quantify the difference in firing rate and spike shape between the transitions from sinusoidal to DC drive.

A comparison of the changes in spike rate under low and high conductance showed a significant difference between the two conditions ( $p<0.0001$, two-way ANOVA; $n=12$ ) (Fig. 9B). Different modulation frequencies under low conductance produced no significant change in mean spike rate versus measures taken with DC drive (difference not significant, Tukey's test; $n=$ 12). Under high conductance, however, sinusoidal modulation of 20 and $30 \mathrm{~Hz}$ produced a substantial increase in spike firing rate relative to no modulation ( $p<0.01$ for 20 and $30 \mathrm{~Hz}$, Tukey's test; $n=12$ ) (Fig. 9B). Frequency modulations beyond $40 \mathrm{~Hz}$ did not significantly increase spike firing rate (difference not significant, Tukey's test; $n=12$ ) (Fig. 9B). This is consistent with filtering caused by the membrane time constant and the impedance profile of pyramidal cells, which showed a substantial drop in impedance at frequencies $>30 \mathrm{~Hz}$ (data not shown).

We also measured the difference in the spike rate of rise as a function of modulation frequency under low and high conductance (Fig. 9C,D). As with spike frequency, the ability for sinusoidal modulation to affect the rate of rise differed significantly between low and high conductance $(p=0.0006$, two-way ANOVA; $n=12$ ) (Fig. 9D). Under low conductance, the difference in the rate of rise between DC drive and sinusoidal modulation did not change significantly for any of the modulation frequencies tested (difference not significant, Tukey's test; $n=$ 12) (Fig. 9D). Under high conductance, modulation frequencies of 10 and $20 \mathrm{~Hz}$ significantly increased the rate of rise compared with DC drive $(p<0.05 ; n=12)$ (Fig. 9D). Like changes in spike firing frequency, the rate of rise also peaked at intermediate sinusoidal modulation frequencies. Consistent with our hypothesis, there exists an optimal frequency range for increasing firing rate under high membrane conductance that is significantly higher than the timescale of spike frequency adaptation in CA1 pyramidal cells. 

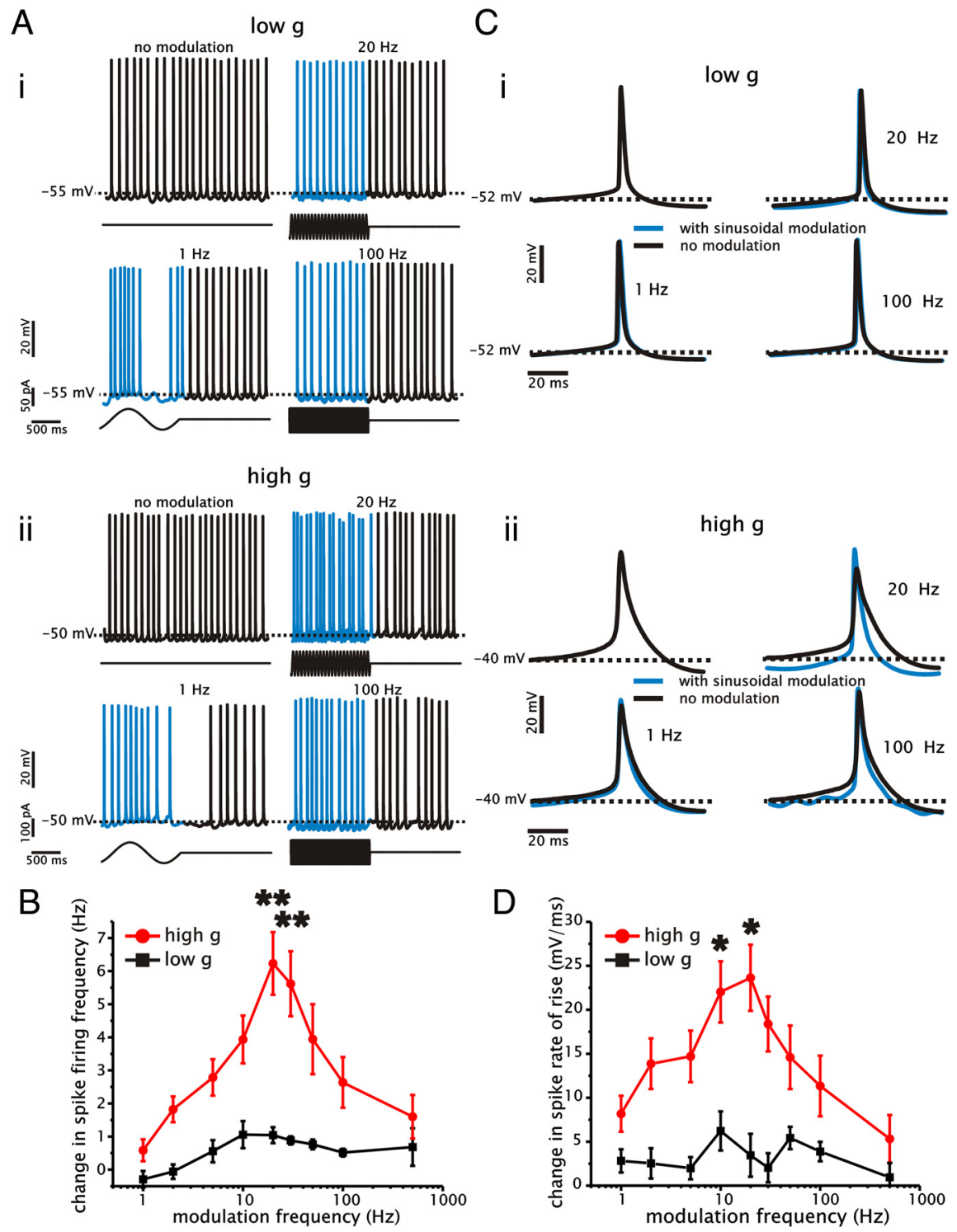

Figure 9. S Sinusoidal modulations of intermediate frequencies preferentially increase spike firing frequency and spike rate of rise under high membrane conductance. $\boldsymbol{A}$, Example voltage traces recorded under low conductance (control conditions) (i) and with high membrane conductance (ii) without modulation (top left), with $1 \mathrm{~Hz}$ (bottom left), $20 \mathrm{~Hz}$ (top right), and $100 \mathrm{~Hz}$ (bottom right) sinusoidal modulation of the input current. The injected input current waveform is displayed below the voltage traces. $\boldsymbol{B}$, Plot of average difference in spike firing frequency as a function of the sinusoidal modulation frequency injected with high (red) and low (black) membrane conductance. C, Example spike-triggered averages of the membrane voltage without (black) and with (blue) sinusoidal modulation of the injected current under low (i) and high conductance (ii). D, Plot of average difference in the spike rate of rise as a function of the sinusoidal modulation frequency injected with (red) and without (black) added conductance. Asterisks indicate significant difference between low conductance and increased conductance: ${ }^{*} p<0.05$; ${ }^{* *} p<0.01$.

\section{Discussion}

The present study demonstrates that membrane voltage fluctuations and conductance interact in a nontrivial manner to shape the input-output relationship of CA1 pyramidal neurons. The present study reports the following novel findings (1) Membrane voltage fluctuations under high conductance restore available $\mathrm{Na}^{+}$ current and preserve output gain relative to high membrane conductance alone. (2) $\mathrm{Na}^{+}$current availability is restored through a hyperpolarization of mean spiking voltage. (3) The hyperpolarization of mean spiking voltage is mediated by two mechanisms. At low firing rates voltage fluctuations allow spiking at mean voltages below the actual spike threshold through probabilistic threshold traversal. At medium to high firing rates, a separation of the timescales of membrane voltage fluctuations and the spike generation mechanism versus the timescale of $\mathrm{Na}^{+}$current inactivation allow spike generation at a lower spiking voltage for a given spike rate. (4) These effects are mediated by voltage fluctuations in a physiologically relevant range of frequencies $(10-40 \mathrm{~Hz})$. (5) Through the restoration of $\mathrm{Na}^{+}$current, hyperpolarizing membrane voltage fluctuations mediated by $\mathrm{GABA}_{\mathrm{A}}$ like inputs can increase firing rate in a highconductance state. Thus, basic integration properties of CA1 pyramidal neurons will depend on interactions between membrane conductance levels and the frequency content and magnitude of voltage fluctuations associated with synaptic activity.

Increased mean membrane conductance depolarizes mean spiking voltage and increases $\mathrm{Na}^{+}$current inactivation

As demonstrated previously, an increase in membrane conductance instantaneously depolarizes the spike voltage threshold in CA1 pyramidal neurons (Prescott et al., 2006; Fernandez and White, 2010). This effect is related to the instantaneous shunting of $\mathrm{Na}^{+}$current by increased membrane conductance. Due to the shunting of $\mathrm{Na}^{+}$ current, the added membrane conductance forces a larger depolarization to activate sufficient $\mathrm{Na}^{+}$current to generate spike firing. Fundamentally, added linear conductance increases the voltage range over which the resting voltage of the neuron (nonspiking behavior) is stable. In CA1 pyramidal neurons, the depolarized spike voltage threshold increases the mean spiking voltage and subsequently causes cumulative $\mathrm{Na}^{+}$current inactivation. The instantaneous depolarization of spike threshold is independent of slow, cumulative $\mathrm{Na}^{+}$current inactivation or spike frequency adaptation, as it depends on an interaction between fast $\mathrm{Na}^{+}$current and membrane leak conductance. Thus, the shift in voltage threshold with conductance is required for increasing cumulative $\mathrm{Na}^{+}$current inactivation and spike frequency adaptation but is not a direct cause of the changes in gain. The reduction in gain requires the cell to have the steady-state and cumulative inactivation properties that enable a sufficient loss of $\mathrm{Na}^{+}$current at steady state with a depolarizing shift in mean spiking voltage.

\section{Two mechanisms are involved in hyperpolarizing mean} spiking voltage and restoring $\mathrm{Na}^{+}$current

Under high conductance, membrane voltage fluctuations increase spike firing rate through two different mechanisms. The first mechanism is well established and increases firing rate only in the lower region of the $f-I$ relationship through transient membrane voltage excursions past spike voltage threshold; volt- 
age fluctuations are causing spikes at mean voltages where no spikes would be generated in the absence of membrane voltage fluctuations (Hô and Destexhe, 2000; Tiesinga et al., 2000; Chance et al., 2002; Hansel and van Vreeswijk, 2002; Miller and Troyer, 2002; Mitchell and Silver, 2003). Through this mechanism, spikes can be generated at mean voltages below the actual voltage threshold. The second mechanism plays a major role in the middle and upper regions of the $f-I$ relationship. It requires a substantial separation of timescales between fast activating variables (e.g., $\mathrm{Na}^{+}$current activation and membrane voltage) and slower dampening variables (e.g., slow $\mathrm{Na}^{+}$current inactivation). This enables membrane voltage and $\mathrm{Na}^{+}$current activation to outpace the slow variable associated with spike frequency adaptation and generate spikes at lower mean voltages. Without this separation of timescales, positive voltage deflections would add spikes, while negative voltage deflections would remove spikes, leading to no net difference between the spike rates with or without voltage fluctuations. Previous models have reproduced this behavior by incorporating variables (e.g., $\mathrm{Na}^{+}$current inactivation or $\mathrm{K}^{+}$current activation) that are slow relative to the timescale of membrane voltage fluctuations (Arsiero et al., 2007; Lundstrom et al., 2009). An important experimental result supporting this mechanism is the unique ability for relatively highfrequency $(>10 \mathrm{~Hz})$ sinusoidal modulations of membrane voltage to increase spike rate when membrane conductance was increased. Consequently, the ability for neuronal input to influence spike output rate will depend on both its frequency content and whether it changes mean membrane conductance. In particular, the frequency range between 10 and $40 \mathrm{~Hz}$, which lies within the theta-gamma range, was found to be most effective at increasing spike rate.

\section{Comparison with previous gain control mechanisms}

Previous modeling and experimental work has emphasized the ability for membrane voltage fluctuations to reduce gain, while changes in membrane conductance alone are thought to lead to subtractive changes in the $f-I$ relationship (Hô and Destexhe, 2000; Tiesinga et al., 2000; Chance et al., 2002; Mitchell and Silver, 2003; Prescott and De Koninck, 2003; Ulrich, 2003). The ability for voltage fluctuations to permit firing rate to scale with subthreshold membrane voltage has been shown to cause a reduction in gain when combined with an increase in membrane conductance (Mitchell and Silver, 2003; Prescott and De Koninck, 2003). Due to the simplicity and few biophysical requirements of this mechanism, it has been suggested that this form of gain control represents a general principle in neuronal processing (Priebe and Ferster, 2002; Destexhe et al., 2003; Haider et al., 2006; Silver, 2010). Although this mechanism likely applies in many cells, our result suggests that it may not be generally applicable to principal cells with slow internal dynamics. Consistent with these conclusions, two recent reports showing either no change or an increase in gain when current-based input fluctuations were used in cortical pyramidal cells expressing substantial spike frequency adaptation (Higgs et al., 2006; Arsiero et al., 2007) but a decrease in the gain of interneurons using the same stimulus (Higgs et al., 2006). Together, these results suggest that changes in conductance and voltage fluctuations may be integrated differently in interneurons and principal cells.

An increase in firing rate throughout the $f-I$ relationship was observed only during steady-state spike generation. The initial $f-I$ relationship of CA1 pyramidal cells behaved largely as predicted by previous studies. The results observed for steady-state firing require $\sim 100$ to $300 \mathrm{~ms}$ to take effect (Fernandez and White,
2010). Furthermore, the detection of gain changes associated with membrane voltage fluctuations requires time to estimate mean spike rate. Thus, the observed changes in steady-state gain described here are not instantaneous but require a certain amount of time to take effect. The noninstantaneous nature of the gain modulation mechanisms described in the present study are also present in other proposed mechanisms. These include various forms of gain control requiring membrane voltage fluctuations or short-term synaptic plasticity (Abbott et al., 1997; Chance et al., 2002; Mitchell and Silver, 2003; Prescott and De Koninck, 2003; Rothman et al., 2009).

\section{Hippocampal inputs in vivo}

Several in vivo studies from sensory cortices have provided experimental evidence for fluctuation-driven spike activity evoked by behaviorally relevant stimuli (Hirsch et al., 1998; Anderson et al., 2000, 2001; Cardin et al., 2008; Ozeki et al., 2009; Gentet et al., 2010). In hippocampus, these issues have received less attention, in part because of technical difficulties and a lack of intracellular in vivo recordings. Like cortical cells, however, intracellular in vivo recordings indicate that CA1 pyramidal cells experience periods of high membrane conductance associated with large membrane voltage fluctuations that drive spike activity between 1 and $15 \mathrm{~Hz}$ (Penttonen et al., 1998; Henze and Buzsáki, 2001; Destexhe et al., 2003; Harvey et al., 2009; Epsztein et al., 2010). In addition, in vitro work on hippocampal oscillations also indicates that significant increases in membrane conductance established by a balance of excitation and inhibition under noisy conditions are involved in spike generation (Atallah and Scanziani, 2009). These studies suggest that the principles of spike generation established in cortex involving large changes in membrane conductance and synaptic-mediated voltage fluctuations also likely apply in the hippocampus.

\section{References}

Abbott LF, Varela JA, Sen K, Nelson SB (1997) Synaptic depression and cortical gain control. Science 275:220-224.

Anderson JS, Lampl I, Gillespie DC, Ferster D (2000) The contribution of noise to contrast invariance of orientation tuning in cat visual cortex. Science 290:1968-1972.

Anderson JS, Lampl I, Gillespie DC, Ferster D (2001) Membrane potential and conductance changes underlying length tuning of cells in cat primary visual cortex. J Neurosci 21:2104-2112.

Arsiero M, Lüscher HR, Lundstrom BN, Giugliano M (2007) The impact of input fluctuations on the frequency-current relationships of layer 5 pyramidal neurons in the rat medial prefrontal cortex. J Neurosci 27:3274-3284.

Atallah BV, Scanziani M (2009) Instantaneous modulation of gamma oscillation frequency by balancing excitation with inhibition. Neuron 62:566-577.

Bettencourt JC, Lillis KP, Stupin LR, White JA (2008) Effects of imperfect dynamic clamp: computational and experimental results. J Neurosci Methods 169:282-289.

Carandini M, Heeger DJ (1994) Summation and division by neurons in primate visual cortex. Science 264:1333-1336.

Cardin JA, Palmer LA, Contreras D (2008) Cellular mechanisms underlying stimulus-dependent gain modulation in primary visual cortex neurons in vivo. Neuron 59:150-160.

Chance FS, Abbott LF, Reyes AD (2002) Gain modulation from background synaptic input. Neuron 35:773-782.

Destexhe A, Rudolph M, Fellous JM, Sejnowski TJ (2001) Fluctuating synaptic conductances recreate in vivo-like activity in neocortical neurons. Neuroscience 107:13-24.

Destexhe A, Rudolph M, Paré D (2003) The high-conductance state of neocortical neurons in vivo. Nat Rev Neurosci 4:739-751.

Dorval AD, Christini DJ, White JA (2001) Real-Time linux dynamic clamp: a fast and flexible way to construct virtual ion channels in living cells. Ann Biomed Eng 29:897-907. 
Epsztein J, Lee AK, Chorev E, Brecht M (2010) Impact of spikelets on hippocampal CA1 pyramidal cell activity during spatial exploration. Science 327:474-477.

Fellous JM, Rudolph M, Destexhe A, Sejnowski TJ (2003) Synaptic background noise controls the input/output characteristics of single cells in an in vitro model of in vivo activity. Neuroscience 122:811-829.

Fernandez FR, White JA (2010) Gain control in CA1 pyramidal cells using changes in somatic conductance. J Neurosci 30:230-241.

Fleidervish IA, Friedman A, Gutnick MJ (1996) Slow inactivation of $\mathrm{Na}+$ current and slow cumulative spike adaptation in mouse and guinea-pig neocortical neurones in slices. J Physiol 493:83-97.

Freund TF, Buzsáki G (1996) Interneurons of the hippocampus. Hippocampus 6:347-470.

Gentet LJ, Avermann M, Matyas F, Staiger JF, Petersen CC (2010) Membrane potential dynamics of GABAergic neurons in the barrel cortex of behaving mice. Neuron 65:422-435.

Haider B, Duque A, Hasenstaub AR, McCormick DA (2006) Neocortical network activity in vivo is generated through a dynamic balance of excitation and inhibition. J Neurosci 26:4535-4545.

Hansel D, van Vreeswijk C (2002) How noise contributes to contrast invariance of orientation tuning in cat visual cortex. J Neurosci 22:5118-5128.

Harvey CD, Collman F, Dombeck DA, Tank DW (2009) Intracellular dynamics of hippocampal place cells during virtual navigation. Nature 461:941-946.

Henze DA, Buzsáki G (2001) Action potential threshold of hippocampal pyramidal cells in vivo is increased by recent spiking activity. Neuroscience 105:121-130.

Higgs MH, Slee SJ, Spain WJ (2006) Diversity of gain modulation by noise in neocortical neurons: regulation by the slow afterhyperpolarization conductance. J Neurosci 26:8787-8799.

Hirsch JA, Alonso JM, Reid RC, Martinez LM (1998) Synaptic integration in striate cortical simple cells. J Neurosci 18:9517-9528.

Hô N, Destexhe A (2000) Synaptic background activity enhances the responsiveness of neocortical pyramidal neurons. J Neurophysiol 84:1488-1496.

Jung HY, Mickus T, Spruston N (1997) Prolonged sodium channel inactivation contributes to dendritic action potential attenuation in hippocampal pyramidal neurons. J Neurosci 17:6639-6646.

Lundstrom BN, Famulare M, Sorensen LB, Spain WJ, Fairhall AL (2009) Sensitivity of firing rate to input fluctuations depends on time scale separation between fast and slow variables in single neurons. J Comput Neurosci 27:277-290.

Maccaferri G, Roberts JD, Szucs P, Cottingham CA, Somogyi P (2000) Cell surface domain specific postsynaptic currents evoked by identified GABAergic neurones in rat hippocampus in vitro. J Physiol 524:91-116.

Mickus T, Jung H, Spruston N (1999) Properties of slow, cumulative so- dium channel inactivation in rat hippocampal CA1 pyramidal neurons. Biophys J 76:846-860.

Miller KD, Troyer TW (2002) Neural noise can explain expansive, powerlaw nonlinearities in neural response functions. J Neurophysiol 87:653-659.

Mitchell SJ, Silver RA (2003) Shunting inhibition modulates neuronal gain during synaptic excitation. Neuron 38:433-445.

Ozeki H, Finn IM, Schaffer ES, Miller KD, Ferster D (2009) Inhibitory stabilization of the cortical network underlies visual surround suppression. Neuron 62:578-592.

Penttonen M, Kamondi A, Acsády L, Buzsáki G (1998) Gamma frequency oscillation in the hippocampus of the rat: intracellular analysis in vivo. Eur J Neurosci 10:718-728.

Prescott SA, De Koninck Y (2003) Gain control of firing rate by shunting inhibition: roles of synaptic noise and dendritic saturation. Proc Natl Acad Sci U S A 100:2076-2081.

Prescott SA, Ratté S, De Koninck Y, Sejnowski TJ (2006) Nonlinear interaction between shunting and adaptation controls a switch between integration and coincidence detection in pyramidal neurons. J Neurosci 26: 9084-9097.

Prescott SA, Ratté S, De Koninck Y, Sejnowski TJ (2008) Pyramidal neurons switch from integrators in vitro to resonators under in vivo-like conditions. J Neurophysiol 100:3030-3042.

Priebe NJ, Ferster D (2002) A new mechanism for neuronal gain control (or how the gain in brains has mainly been explained). Neuron 35:602-604.

Priebe NJ, Ferster D (2008) Inhibition, spike threshold, and stimulus selectivity in primary visual cortex. Neuron 57:482-497.

Rothman JS, Cathala L, Steuber V, Silver RA (2009) Synaptic depression enables neuronal gain control. Nature 457:1015-1018.

Shu Y, Hasenstaub A, Badoual M, Bal T, McCormick DA (2003) Barrages of synaptic activity control the gain and sensitivity of cortical neurons. J Neurosci 23:10388-10401.

Silver RA (2010) Neuronal arithmetic. Nat Rev Neurosci 11:474-489.

Slee SJ, Higgs MH, Fairhall AL, Spain WJ (2005) Two-dimensional time coding in the auditory brainstem. J Neurosci 25:9978-9988.

Tiesinga PH, José JV, Sejnowski TJ (2000) Comparison of current-driven and conductance-driven neocortical model neurons with HodgkinHuxley voltage-gated channels. Phys Rev E Stat Phys Plasmas Fluids Relat Interdiscip Topics 62:8413-8419.

Treue S, Martínez Trujillo JC (1999) Feature-based attention influences motion processing gain in macaque visual cortex. Nature 399:575-579.

Ulrich D (2003) Differential arithmetic of shunting inhibition for voltage and spike rate in neocortical pyramidal cells. Eur J Neurosci 18:2159-2165.

Yau HJ, Baranauskas G, Martina M (2010) Flufenamic acid decreases neuronal excitability through modulation of voltage-gated sodium channel gating. J Physiol 588:3869-3882. 